CERN-PH-TH-2012-189

UCB-PTH-12/11

\title{
ABCD of Beta Ensembles and Topological Strings
}

\author{
Daniel Krefl ${ }^{a}$ and Johannes Walcher ${ }^{b}$ \\ a Center for Theoretical Physics \\ University of California, Berkeley, California, USA \\ ${ }^{b}$ Departments of Physics, and Mathematics and Statistics \\ McGill University, Montréal, Québec, Canada
}

\begin{abstract}
We study $\beta$-ensembles with $B_{N}, C_{N}$, and $D_{N}$ eigenvalue measure and their relation with refined topological strings. Our results generalize the familiar connections between local topological strings and matrix models leading to $A_{N}$ measure, and illustrate that all those classical eigenvalue ensembles, and their topological string counterparts, are related one to another via various deformations and specializations, quantum shifts and discrete quotients. We review the solution of the Gaussian models via Macdonald identities, and interpret them as conifold theories. The interpolation between the various models is plainly apparent in this case. For general polynomial potential, we calculate the partition function in the multi-cut phase in a perturbative fashion, beyond tree-level in the large- $N$ limit. The relation to refined topological string orientifolds on the corresponding local geometry is discussed along the way.
\end{abstract}

July 2012 


\section{Contents}

1 Introduction $\quad 2$

2 Macdonald ensembles 6

3 Gaussian ensembles: Large $N$ partition functions

3.1 Large $N$ expansions . . . . . . . . . . . . . . . . . . . . . . . 8

3.2 Implications for toric Calabi-Yau backgrounds . . . . . . . . . . . . . 13

4 Gaussian ensembles: Correlators

5 Multi-cut potentials: Perturbative calculation $\quad 17$

5.1 Saddle-point approximation ................... . . 18

5.2 Examples: $B_{N}$ and $D_{N}$ quartic ................ . . 20

6 B-model verification of $B_{N}$ and $D_{N}$ quartic $\quad 23$

6.1 Tree-level geometry . . . . . . . . . . . . . . . . . . 23

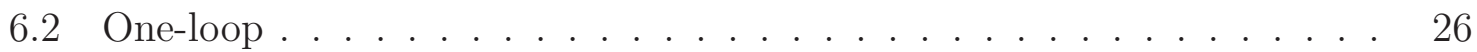

7 Conclusion $\quad 27$

$\begin{array}{ll}\text { Acknowledgments } & 28\end{array}$

A Free energies $\quad 29$

References

\section{Introduction}

Eigenvalue ensembles with $A_{N}$ measure to a power of $\beta, 1$ widely known just as $\beta$ ensembles, and their relation to topological gauge and string theories have been studied extensively in recent years. The special instance $\beta=1$ of generalized interest is the Dijkgraaf-Vafa relation [1] between matrix models, supersymmetric gauge theory and the topological string. In more recent times, the focus has shifted to the more general situation with arbitrary $\beta$, which relates the eigenvalue ensembles to $\Omega$-deformed gauge theories, refined topological string theory [2, 3] and the AGT conjecture [4]. Here, the

\footnotetext{
${ }^{1} A_{N}$ denotes the finite Coxeter group, and $\beta$ a positive real number. We review the definitions in section 2
} 
equivariant parameters $\epsilon_{i}$ of the $\Omega$-deformation, the ensemble parameter $\beta$ and the string coupling $g_{s}$ are related via [2],

$$
\epsilon_{1}=\sqrt{\beta} g_{s}, \quad \epsilon_{2}=-\frac{1}{\sqrt{\beta}} g_{s} .
$$

One may note that neither the matrix model nor the topological string at present knows a microscopic interpretation for the deformation parameter $\beta$. Rather, the mutual agreement of results calculated with different schemes, the consistency of the space-time interpretation via BPS state/instanton counting, as well as the relation with the $\Omega$-deformed gauge theory, especially in the Nekrasov-Shatashvili limit [5] give confidence that one should view all these models as integral part of a larger interconnected web of theories, thereby in fact defining various notions of quantum geometry, such as that of [3].

The prototypical example for much of this is the Gaussian model, with quadratic potential for the eigenvalues and corresponding, respectively, to a deformed conifold target space, (refined) Chern-Simons theory [6], as well as the $c=1$ non-critical string at radius $R=\beta$ [7, 2]. This Gaussian model also serves as building block for more general backgrounds.

The purpose of the present paper is to take this logic one step further, and to study the possible role played by eigenvalue ensembles with other finite group measures, specifically, $B_{N}, C_{N}$, and $D_{N}$. These models, which we will refer to as Macdonald ensembles, are rather natural, and easily defined, but have been less studied in the recent topological string/gauge theory literature. The ensembles at $\beta=1$ appeared briefly in the context of the Dijkgraaf-Vafa relation to four-dimensional $\mathcal{N}=1$ gauge theories with $S O / S p$ gauge groups and adjoint matter, and the realization of these gauge theories as string theoretic orientifolds. Most closely related to the spirit of the present work are [8] and [9]. Due to the nature of the original DV conjecture, these studies were essentially confined to tree-level. One of the aims of this work is to study the $B_{N} / C_{N}$ and $D_{N}$ eigenvalue ensembles with general $\beta$ beyond tree-level in greater detail 2

It is then natural to expect that the $B_{N}$, and $D_{N}$ Macdonald ensembles with general $\beta \neq 1$ are related to a refinement of topological string orientifolds, which was one of

\footnotetext{
${ }^{2}$ Since the root systems of $B_{N}$ and $C_{N}$ differ only in the length of the roots, hence the Haar measures are identical up to an overall factor (see for instance [10]), it will be sufficient for us to consider only the $B_{N}$ and $D_{N}$ ensembles.
} 
the original motivations for the present work 3 In thinking about the various pictures, it is however important to remember that these eigenvalue ensembles at $\beta=1$ are in general not identical to the usual $S O$ and $S p$ matrix models. Rather, the latter models provide the microscopic realization of the $A_{N}$ ensemble at $\beta=2$ and $\beta=$ $1 / 2$, respectively. They are dual to $\mathcal{N}=1 S O / S p$ gauge theory with matter in the symmetric/antisymmetric representation. In particular, the orientifold in the large- $N$ dual topological string side acts differently on the tree-level geometry [12, 13].

On the other hand we have the realization, in the Gaussian model, of the $\beta$ parameter as the radius of the circle for $c=1$ non-critical string. There, the orbifold of the $c=1 \mathrm{CFT}$ at the self-dual radius is indeed equivalent to the $R=2$ circle theory. This connection suggests the existence of an entire new branch of topological string/matrix model dual pairs that connects up to the standard branch at $\beta=2$. Our work suggests that this is where the $B_{N}$ and $D_{N}$ Macdonald ensembles fit in.

Whereas the duality between $\mathcal{N}=2 U(N)$ gauge theory softly broken to $\mathcal{N}=1$, $A_{N}$ eigenvalue ensemble with $\beta=1$, and topological string theory on the (spectral curve) geometry at large $N$ has been discussed and checked exhaustively in many works, for general $\beta$ much less is known. For $B_{N}$ and $D_{N}$, even at $\beta=1$, no higher genus check of the proposed duality between the eigenvalue ensemble and topological string orientifolds has been performed. The power of $\beta$ plays a major role beyond treelevel, and hence one might hope to be able to learn something about refined topological string theory and orientifolds thereof along the way, which are expected to be related to these $\beta$-ensembles in the large $N$ limit.

In fact, under which specific conditions the eigenvalue ensembles for $\beta \neq 1$ relate to refined topological string theory in the large $N$ limit has not been pointed out so far in the literature, even for the ordinary $A_{N}$ measure. Some examples where such a relation holds in a non-trivial manner where reported in [2, 3]. Although the calculations of [3] were restricted to the cubic ensemble at 1-loop level, there is little doubt that the observed correspondence extends to all genus, at least for the cubic. On the other hand, the Chern-Simons matrix models studied in [14] appear to indicate that in general such a relation does not hold. Attempts to formulate a refined version of the remodeled B-model of [15] have also failed to our knowledge so far.

Some of the problems with the general applicability of the $A_{N}$ type $\beta$-ensemble can

\footnotetext{
${ }^{3}$ Meanwhile, the refinement of topological string orientifolds has been studied, with a different perspective and motivation, by Aganagic and Schaeffer [11].
} 
be traced back all the way to tree-level, that is, to the dual spectral curve geometry of the ensemble. This is most clearly visible at hand of the remodeled B-model geometries of [16]: In general, the spectral curve of the eigenvalue ensemble differs from the usual B-model target space geometry of the dual topological string and has singular points. Singularities are a general indication that refinement, i.e., a deformation of the correspondence away from $\beta=1$, will fail. Indeed, singularities in the B-model geometry could harbor blow-up modes, which spoil an invariant BPS state counting. The corresponding mirror statement is the well-known fact that in order to have a well-defined BPS state counting of left and right spin (and not just the index), the A-model/M-theory geometry should be rigid (i.e., have no complex structure deformations). This leads us to a condition on an $A_{N}$ type $\beta$-ensemble to have a well-defined BPS state counting interpretation. Namely, the spectral curve has to be non-singular. In particular, this applies as well to ensembles with polynomial potentials, i.e., one has to fill all cuts to ensure that one has a well defined BPS index. Under this restriction, the duality of [2] has a chance to survive the $\beta$-deformation in a quite general setting. Similar considerations apply to the $B_{N}$ and $D_{N}$ cases, up to some technicality which we will explain in more detail in section 5.1. Confirmation for this expectation will be found at hand of $A_{N}, B_{N}$ and $D_{N} \beta$-ensembles with quartic potential, which appear to be as well compatible with a (refined) topological string interpretation, as the free energies fulfill the 1-loop holomorphic anomaly equation.

The outline is as follows. In section 2 we will give the definition of Macdonald ensembles with special emphasize on $A_{N}, B_{N}$ and $D_{N}$. This is followed by a detailed discussion of the large $N$ expansion of the Gaussian partition functions and implications thereof for refined topological string orientifolds, in section 3, In section 4 a recursion relation satisfied by Gaussian correlators is derived (generalizing [17, 18]), which constitute an essential ingredient for the explicit calculation of the multi-cut ensemble partition function, which section 5 is about. In subsection 5.1 , we will give a generalization of the framework of [19, 20, 17, 18] to $B_{N} / D_{N}$, and apply it in section 5.2 to the model with quartic potential. The B-model verification of the tree-level and 1-loop results of section 5.2 will be performed in section 6 . We conclude in section 7 . In appendix $\mathrm{A}$ the explicit results for the $g_{s}$ expansion of the free energy of the $B_{N}$ and $D_{N} \beta$-ensemble with quartic potential are attached. 


\section{Macdonald ensembles}

Let $\mathcal{G}$ be a finite group of isometries of $\mathbb{R}^{N}$ generated by reflections in hyperplanes through the origin (i.e., a finite reflection or Coxeter group). Let there be $h$ hyperplanes, each defined by a condition on $\lambda \in \mathbb{R}^{N}$ of the form

$$
\sum_{i=1}^{N} a_{\alpha, i} \lambda_{i}=0
$$

where $a_{\alpha} \in \mathbb{R}^{N}, \alpha=1, \ldots, h$. The group $\mathcal{G}$ naturally acts on the algebra of polynomial functions on $\mathbb{R}^{N}$. The $\mathcal{G}$-invariant polynomials form an $\mathbb{R}$-algebra generated by $N$ independent polynomials of degrees $d_{i}, i=1, \ldots, N$. Normalizing the vectors $\left(a_{\alpha, i}\right)_{i}$ via $\sum_{i=1}^{N} a_{\alpha, i}^{2}=2$, define the particular invariant polynomial

$$
P_{\mathcal{G}}(\lambda)=\prod_{\alpha=1}^{h} \sum_{i=1}^{N} a_{\alpha, i} \lambda_{i}
$$

Macdonald conjectured the integral identity [21]

$$
Z_{\mathcal{G}}(\beta):=\frac{1}{(2 \pi)^{N / 2}} \int_{\mathbb{R}^{N}}[d \lambda]\left|P_{\mathcal{G}}(\lambda)\right|^{2 \beta} e^{-\frac{1}{2} \sum_{i=1}^{N} \lambda_{i}^{2}}=\prod_{i=1}^{N} \frac{\Gamma\left(1+d_{i} \beta\right)}{\Gamma(1+\beta)},
$$

where $[d \lambda]:=\prod_{i=1}^{N} d \lambda_{i}, \beta \in \mathbb{C}$ with $\operatorname{Re} \beta>0$. A proof of this identity has been given by Opdam [22, 23]. $Z_{\mathcal{G}}(\beta)$ is also referred to as Macdonald integral.

For $\mathcal{G}=A_{N-1}$ the Macdonald integral specializes to Mehta's integral. For this work, in addition the cases $\mathcal{G}=B_{N}$ and $\mathcal{G}=D_{N}$ are of particular interest. These give rise to the integral identities,

$$
\begin{aligned}
Z_{A_{N-1}}(\beta) & :=\frac{1}{(2 \pi)^{N / 2}} \int_{\mathbb{R}^{N}}[d \lambda] \Delta(\lambda)^{2 \beta} e^{-\frac{1}{2} \sum_{i} \lambda_{i}^{2}}=\prod_{i=1}^{N} \frac{\Gamma(1+i \beta)}{\Gamma(1+\beta)}, \\
Z_{B_{N}}(\beta) & :=\frac{1}{(2 \pi)^{N / 2}} \int_{\mathbb{R}^{N}}[d \lambda] \Delta\left(\lambda^{2}\right)^{2 \beta} \prod_{i=1}^{N} \lambda_{i}^{2 \beta} e^{-\frac{1}{2} \sum_{i} \lambda_{i}^{2}}=\prod_{i=1}^{N} \frac{\Gamma(1+2 i \beta)}{\Gamma(1+\beta)}, \\
Z_{D_{N}}(\beta) & :=\frac{1}{(2 \pi)^{N / 2}} \int_{\mathbb{R}^{N}}[d \lambda] \Delta\left(\lambda^{2}\right)^{2 \beta} e^{-\frac{1}{2} \sum_{i} \lambda_{i}^{2}}=\frac{\Gamma(1+N \beta)}{\Gamma(1+\beta)} \prod_{i=1}^{N-1} \frac{\Gamma(1+2 i \beta)}{\Gamma(1+\beta)},
\end{aligned}
$$

where $\Delta(\lambda)$ denotes the usual Vandermonde determinant, $\Delta(\lambda):=\prod_{i<j}\left(\lambda_{i}-\lambda_{j}\right)$. We are particularly interested in the large $N$ limit thereof, see section 3 ,

Viewing the above integrals as partition functions of Gaussian eigenvalue ensembles, it is natural to define general Macdonald ensembles by replacing the quadratic term 
$\sum_{i} \lambda_{i}^{2}$ with a general "single-trace" polynomial potential $\sum_{i} W\left(\lambda_{i}\right)$. For $\mathcal{G}=A_{N-1}$ the Macdonald ensemble is identical to the usual $\beta$-ensemble. For $\mathcal{G}=B_{N}$ and $D_{N}$, and with $\beta=1$, these ensembles correspond to the ones considered in [8] in the context of the Dijkgraaf-Vafa relation (with the additional condition $W(x)=W(-x)$ ).

It is convenient to parameterize the measure $P_{(b, d)}(\lambda):=P_{\mathcal{G}}(\lambda)$ with $\mathcal{G}$ being $A_{N}$, $B_{N}$ or $D_{N}$ as

$$
P_{(b, d)}(\lambda)=\Delta_{+}(\lambda)^{b+d} \Delta_{-}(\lambda) \prod_{i=1}^{N} \lambda_{i}^{b},
$$

where we defined

$$
\Delta_{ \pm}(\lambda):=\prod_{i<j}^{N}\left(\lambda_{i} \pm \lambda_{j}\right) .
$$

In particular $\Delta_{-}(\lambda)=\Delta(\lambda)$, corresponds to the usual Vandermonde, and $\Delta_{-}(\lambda) \Delta_{+}(\lambda)=$ $\Delta\left(\lambda^{2}\right)$. For $(b, d)=(0,0)$ we get $P_{A_{N}}(\lambda),(1,0)$ yields $P_{B_{N}}(\lambda)$ and $(0,1)$ results in $P_{D_{N}}(\lambda)$. Hence the Macdonald ensembles $Z_{\mathcal{G}}(\beta)$ with $\mathcal{G}=A_{N}, \mathcal{G}=B_{N}$ or $\mathcal{G}=D_{N}$ can be treated simultaneously via the ensemble 4

$$
Z_{(b, d)}(\beta) \sim \int[d \lambda]\left|P_{(b, d)}(\lambda)\right|^{2 \beta} e^{-\sum_{i=1}^{N} W\left(\lambda_{i}\right)}
$$

with $P_{(b, d)}(\lambda)$ as defined in (2.3).

The expectation value for an operator insertion $\hat{\mathcal{O}}$ is defined as usual as

$$
\langle\hat{\mathcal{O}}\rangle_{(b, d)}:=\int[d \lambda]\left|P_{(b, d)}(\lambda)\right|^{2 \beta} \hat{\mathcal{O}} e^{-\sum_{i=1}^{N} W\left(\lambda_{i}\right)}
$$

Trivially, we have $\langle 1\rangle_{(b, d)}=Z_{(b, d)}(\beta)$.

It is instructive to compare the formulas for $B_{N}$ and $D_{N}$. The only difference is the additional factor of $\prod_{i=1}^{N} \lambda_{i}^{2 \beta}$ for $B_{N}$, and can be interpreted as follows. We know from the $A_{N} \beta$-ensemble that the insertion of a brane at position $x$ in the dual geometry corresponds to the insertion of some power of a determinant factor of $\prod_{i=1}^{N}\left(x-\lambda_{i}\right)$ times an overall classical piece of $\psi_{c l}(x)=e^{W(x)}$ [24, 3]. Different powers of the insertion correspond to different types of branes [3]. Thus, the insertion of a brane at $x$ plus a mirror brane at $-x$ corresponds to an operator insertion of

$$
\hat{\Psi}_{\beta}(x) \hat{\Psi}_{\beta}(-x)=\psi_{c l}(x) \psi_{c l}(-x) \prod_{i=1}^{N}\left(\lambda_{i}^{2}-x^{2}\right)^{\beta} .
$$

\footnotetext{
${ }^{4}$ The $g_{s}$ dependence needed to match to topological strings will be brought in via $W(x)$ and, if necessary, a rescaling of the eigenvalues.
} 
This implies that the $B_{N}$ ensemble can be understood as the $D_{N}$ ensemble with insertion of an additional pair of branes at the origin $(x=0)$, i.e.,

$$
Z_{(1,0)}(\beta) \sim\left\langle\hat{\Psi}_{\beta}(0) \hat{\Psi}_{\beta}(0)\right\rangle_{(0,1)}=Z_{(0,1)}(\beta) \Psi_{(0,1)}^{2 \beta}(0),
$$

where we defined the partition function with $h$ coincident $(\beta-)$ branes $\Psi_{(b, d)}^{h \beta}(x)$ in the background parameterized by $(b, d)$ as

$$
\Psi_{(b, d)}^{h \beta}(x):=\frac{\left\langle\left(\hat{\Psi}_{\beta}(x)\right)^{h}\right\rangle_{(b, d)}}{\langle 1\rangle_{(b, d)}} .
$$

\section{Gaussian ensembles: Large $N$ partition functions}

In this section, we review in some detail the large- $N$ expansions of the Gaussian partition functions (2.2), as well as the various ways that these enter into the topological string.

\subsection{Large $N$ expansions}

$A_{N}$

In contrast to the $B_{N}$ and $D_{N}$ ensembles, the 't Hooft large- $N$ limit of $Z_{A_{N-1}}(\beta)$ has been studied extensively in the physics literature. The asymptotic expansion as $N \rightarrow \infty$ is related to the "Schwinger" integral,

$$
\log Z_{A_{N-1}}(\beta) \sim \int \frac{d t}{t} \frac{e^{-\mu t}}{\left(e^{\epsilon_{1} t}-1\right)\left(e^{\epsilon_{2} t}-1\right)}=: \log Z_{A}\left(g_{s}, \beta\right),
$$

where $\epsilon_{1}, \epsilon_{2}$ are related to $g_{s}, \beta$ as in (1.1), and we have

$$
\mu:=\sqrt{\beta} g_{s} N
$$

We note the obvious symmetry of the partition function under $\epsilon_{1} \leftrightarrow \epsilon_{2}$. As $g_{s} \rightarrow 0$, we have the well-known asymptotic expansion

$$
\int \frac{d t}{t} \frac{e^{-\mu t}}{\left(e^{\epsilon_{1} t}-1\right)\left(e^{\epsilon_{2} t}-1\right)} \sim \sum_{n=0}^{\infty} \Phi_{A}^{(n)}(\beta)\left(\frac{g_{s}}{\mu}\right)^{n}
$$

with certain polynomial expressions $\Phi_{A}^{(n)}(\beta)$. 5

\footnotetext{
${ }^{5}$ Really, $\beta^{n} \Phi_{A}^{(n)}$ is a polynomial in $\beta$.
} 
As is well-known, for $n$ even, the $\Phi_{A}^{(n)}(\beta)$ specialize at $\beta=1$ to give the virtual Euler characteristic of the moduli space $\mathcal{M}_{g}$ of genus $g=\frac{n}{2}+1$ complex curves [25],

$$
\Phi_{A}^{(2 g-2)}(1)=\chi\left(\mathcal{M}_{g}\right)=\frac{B_{2 g}}{2 g(2 g-2)}
$$

where $B_{n}$ are the Bernoulli numbers. For $n$ odd, the $\Phi_{A}^{(n)}(1)$ vanish.

On the other hand, for $n$ odd, the $\Phi_{A}^{(n)}(\beta)$ specialize at $\beta=2$ to give the virtual Euler characteristic of the moduli space $\mathcal{M}_{\tilde{g}}^{O}$ of complex curves of genus $\tilde{g}=n+1$ with a fixed-point free anti-holomorphic involution (i.e., certain type of real curves) [26, 27, 28], up to a rescaling of $g_{s}$. In string theory language, the quotients give unoriented Riemann surfaces with genus $g=\tilde{g} / 2$, ( $\tilde{g}$ being even), no boundaries, and one crosscap,

$$
\Phi_{A}^{(2 g-1)}(2)=-2^{1 / 2-g} \chi\left(\mathcal{M}_{\tilde{g}}^{O}\right)=-2^{1 / 2-g} \frac{\left(2^{2 g-2}-2^{-1}\right) B_{2 g}}{2 g(2 g-1)}
$$

For $n$ even, we have that $\Phi_{A}^{(2 g-2)}(2)=2^{-g} \chi\left(\mathcal{M}_{g}\right)$. Hence, the coefficients at $\beta=2$ show the typical structure of an orientifold

$$
2^{n / 2} \Phi_{A}^{(n)}(2)=\frac{1}{2} \chi\left(\mathcal{M}_{g}\right)-\chi\left(\mathcal{M}_{\tilde{g}}^{O}\right)
$$

For later reference, note that via making use of (3.1) and (3.6), one can infer as well an integral representation of the generating function for the $\chi\left(\mathcal{M}_{\tilde{g}}^{O}\right)$, i.e.,

$$
\mathcal{T}\left(g_{s}\right):=\log \frac{Z_{A}\left(\sqrt{2} g_{s}, 2\right)}{\sqrt{Z_{A}\left(g_{s}, 1\right)}}=-\frac{1}{2} \int \frac{d t}{t} \frac{e^{-\mu t}}{e^{g_{s} t}-e^{-g_{s} t}} \sim \sum_{n=0}^{\infty} \chi\left(\mathcal{M}_{2 n}^{O}\right)\left(\frac{g_{s}}{\mu}\right)^{2 n-1} .
$$

A similar, and in fact related, "Schwinger" integral has appeared before in the context of the orientifold constant map contribution [29, 30].

The fact that the $A_{N} \beta$-ensemble can be used to interpolate between the Euler characteristic of moduli spaces of complex and real curves was pointed out in [27], and interpreted as a geometric parameterization. In particular, it was conjectured that the $\Phi_{A}^{(n)}(\beta)$ themselves should describe the Euler characteristic of some related moduli space.

Although the appearance of the moduli of real curves is suggestive, a simple closed string theory interpretation is hampered by the fact that the expansion (3.3) for $\beta \neq 1$ contains terms of both even and odd powers of $g_{s}$. This originates from the fact that 
(3.1) is not invariant under $\left(\epsilon_{1}, \epsilon_{2}\right) \rightarrow\left(-\epsilon_{1},-\epsilon_{2}\right)$, except when $\epsilon_{1}=-\epsilon_{2}$. As is by now well-appreciated, the additional (quantum) shift

$$
\mu \rightarrow \mu+\frac{\epsilon_{1}+\epsilon_{2}}{2}
$$

restores that symmetry. We have the asymptotic expansion

$$
\int \frac{d t}{t} \frac{e^{-\mu t}}{\left(e^{\epsilon_{1} t / 2}-e^{-\epsilon_{1} t / 2}\right)\left(e^{\epsilon_{2} t / 2}-e^{-\epsilon_{2} t / 2}\right)} \sim \sum_{n} \Psi_{A}^{(n)}(\beta)\left(\frac{g_{s}}{\mu}\right)^{n}
$$

with $\Psi_{A}^{(n)}(\beta) \equiv 0$ for $n$ odd. This shifted partition function is also identical to the partition function of the $c=1$ string at radius $R \propto \beta$, originally found in [31]. From the above formulas it is clear that $\Phi_{A}^{(n)}(1)=\Psi_{A}^{(n)}(1)$.

Turning to the topological string, it was discovered long time ago in [7], that the integral (3.1) at $\beta=1$, i.e., the $c=1$ string at the self-dual radius, governs the leading behavior of the B-model topological string in the limit in which the target space develops a conifold singularity, as the complex structure parameter $\mu \rightarrow 0$. As explained for instance in [32], the coefficients $\Phi_{A}^{(n)}(1)=\Psi_{A}^{(n)}(1)$ therefore provide universal boundary condition for solving the topological string via holomorphic anomaly equation [33]. As shown in [34, 35], see also [36], the one-parameter deformation $\Psi_{A}^{(n)}(\beta)$ provides the analogous boundary conditions for solving the refined topological string in the Bmodel via the same holomorphic anomaly equation. (Alternatively, one may use the extended holomorphic anomaly equation of [37] with boundary conditions provided by the $\Phi_{A}^{(n)}(\beta)$ to solve for the refined topological string amplitudes after undoing the quantum shift.) This observation confirms the identification of $\beta$ as the radius $R$ of $c=1$ string [2].

A seemingly unrelated observation is the fact that the coefficients $\Psi_{A}^{(n)}(2)$ also have a topological string interpretation, in the context of the real topological string [38]. Namely, writing [34],

$$
2^{n / 2} \Psi_{A}^{(n)}(2)=\frac{1}{2}\left(\Psi_{A}^{(n)}(1)+\Psi_{\mathrm{KB}}\right),
$$

the $\Psi_{\mathrm{KB}}$ control the leading behavior of the topological string amplitude on a genus $g$ Klein-bottle (an unoriented Riemann surface with genus $g$ and even number of crosscaps) around a conifold point in moduli space. This relation begs for a topological interpretation of the $\Psi_{\mathrm{KB}}$ similar to that of the $\Psi_{A}^{(n)}(1)$ in terms of the moduli space of genus $g$ complex curves (with $n=2 g-2$ ). We note that whatever this interpretation is, $\Psi_{A}^{(n)}(1)$ is not the virtual Euler characteristic of moduli of complex curves 
with fixed-point free anti-holomorphic involution of odd genus $\tilde{g}=2 g-1$ (which are the covers of these higher genus Klein bottles) studied in [27], which vanishes, but should be closely related to it. It is also interesting to note that the quantum shift (3.8) transforms Klein-bottle contributions into cross-cap contributions, as is apparent via comparing (3.6) and (3.10).

$B_{N}$

It follows from (2.2) that $Z_{B_{N}}(\beta)$ can be expressed in terms of $Z_{A_{N-1}}(\beta)$ as

$$
\log Z_{B_{N}}(\beta)=\log Z_{A_{N-1}}(2 \beta)+N \log \frac{\Gamma(1+2 \beta)}{\Gamma(1+\beta)} .
$$

Since in our 't Hooft limit, we neglect the most singular terms, of positive power of $N$, we simply write,

$$
Z_{B}\left(g_{s}, \beta\right) \sim Z_{A}\left(g_{s}, 2 \beta\right)
$$

As a result, the $\beta \leftrightarrow 1 / \beta$ symmetry of $Z_{A}(\beta)$ translates to a $\beta \leftrightarrow 1 /(4 \beta)$ symmetry of the $Z_{B}(\beta)$ partition function. Similarly as in the $A_{N}$ case, we denote the expansion coefficients of the $g_{s}$ expansion of the corresponding (shifted) free energy as $\Psi_{B}^{(n)}(\beta)$. Obviously,

$$
\Psi_{B}^{(n)}(\beta)=\Psi_{A}^{(n)}(2 \beta)
$$

For later reference, let us explicitly state the "1-loop" coefficient which the (shifted) $Z_{B}\left(g_{s}, \beta\right)$ implies, i.e.,

$$
\Psi_{B}^{(0)}(\beta)=\frac{1}{48}\left(\frac{1}{\beta}+4 \beta\right)
$$

$D_{N}$

For the $D_{N}$ Macdonald ensemble, we sort terms such that

$$
\log Z_{D_{N}}(\beta / 2)=\log Z_{A_{N-1}}(\beta)-\log \frac{\Gamma(1+N \beta)}{\Gamma(1+N \beta / 2)}+N \log \frac{\Gamma(1+\beta)}{\Gamma(1+\beta / 2)} .
$$

Thus, besides the $A_{N}$ term, we have one additional $N$ dependent term which we don't neglect in the large- $N$ limit. Invoking the integral representation of the digamma function $\gamma(x)=\frac{d}{d x} \log (\Gamma(x))$,

$$
\gamma(x)=\int_{0}^{\infty} d t\left(\frac{e^{-t}}{t}-\frac{e^{-x t}}{1-e^{-t}}\right)
$$


one can infer that the essential part of the new contribution reads

$$
-\int \frac{d t}{t} \frac{e^{-N \beta t}-e^{-N \beta t / 2}}{1-e^{-t}}
$$

Redefining $t \rightarrow t \frac{g_{s}}{\sqrt{\beta}}$ and taking $N \rightarrow \infty$ while keeping $\sqrt{\beta} N g_{s}=: \mu$ fixed yields

$$
-\int \frac{d t}{t} \frac{e^{-\mu t}-e^{-\mu t / 2}}{1-e^{-\frac{g_{s}}{\sqrt{\beta}} t}}
$$

Reverting to our usual notation, we obtain (up to non-universal terms)

$$
\log Z_{D_{N}}(\beta / 2) \sim \int \frac{d t}{t} \frac{e^{-\mu t}}{\left(e^{\epsilon_{1} t}-1\right)\left(e^{\epsilon_{2} t}-1\right)}+\int \frac{d t}{t} \frac{e^{-\mu t}}{e^{\epsilon_{2} t}-e^{-\epsilon_{2} t}}=: \log Z_{D}\left(g_{s}, \beta / 2\right) .
$$

We recognize the second term as being essentially the generating function (3.7). Hence, $Z_{D}\left(g_{s}, \beta\right)$ is entirely given by a combination of $Z_{A}$, i.e.,

$$
\log Z_{D}\left(g_{s}, \beta\right)=\log Z_{A}\left(g_{s}, 2 \beta\right)-2 \mathcal{T}\left(\sqrt{2 \beta} g_{s}\right) .
$$

(where $\mathcal{T}$ is defined in (3.7).) It is also instructive to express the "1-loop" coefficient $\Phi_{D}^{(0)}(\beta)$ contained in (3.14) in terms of $\Phi_{A}^{(0)}(\beta)$. Using the relations (3.15) and (3.7), we infer

$$
\Phi_{D}^{(0)}(\beta)=\Phi_{A}^{(0)}(2 \beta)+2\left(\Phi_{A}^{(0)}(2)-\frac{1}{2} \Phi_{A}^{(0)}(1)\right) .
$$

We observe that this result matches the structure of the orbifold branch partition function for the $c=1 \mathrm{CFT}$ on the torus derived in [39]. We take this as a hint that (3.15) is related to the partition function of the orbifold branch of the $c=1$ non-critical string. More precisely, such a relation should hold after an appropriate quantum shift. One way to identify the appropriate $D_{N}$-analog of (3.8) is to impose a symmetry under $g_{s} \rightarrow-g_{s}$. The symmetry can be motivated as follows. We know that for integer values of $\beta$ the partition function of the $c=1$ string on the circle branch can be matched to the partition function of the topological string expanded near a $A_{\beta-1}$ type singularity (under appropriate choice of deformation parameters) 40]. Since the chiral ground ring manifold of the $\mathrm{c}=1$ string on the orbifold branch corresponds to a Kleinian singularity of $D$-type [41], we expect that similarly the orbifold branch partition function can be matched to the topological string expanded near a $D$-type singularity, implying the symmetry under $g_{s} \rightarrow-g_{s}$.

Indeed, after

$$
\mu \rightarrow \mu-\frac{\epsilon_{2}}{2}
$$


the free energy (3.14) becomes

$$
\int \frac{d t}{t} \frac{e^{-\mu t} \cosh \left(\frac{\left(\epsilon_{1}+\epsilon_{2}\right) t}{2}\right)}{2 \sinh \left(\epsilon_{1} t / 2\right) \sinh \left(\epsilon_{2} t\right)} \sim \sum_{n} \Psi_{D}^{(n)}(\beta / 2)\left(\frac{g_{s}}{\mu}\right)^{n}
$$

and clearly possesses an even power only expansion in $g_{s}$. 6 We denote the expansion coefficients by $\Psi_{D}^{(n)}(\beta)$. For the special value $\beta=1$, we find in addition

$$
\Psi_{D}^{(n)}(1)=\Psi_{B}^{(n)}(1)=\Psi_{A}^{(n)}(2)
$$

This identity is precisely the one expected from the matching of circle and orbifold branches of $c=1$ string at $R=1$ and $R=2$, respectively. It is important to note however that generally, $Z_{A_{N}}$ and $Z_{D_{N}}$ are not related by a simple shift of $N$.

For later reference, we explicitly state the "1-loop" coefficient

$$
\Psi_{D}^{(0)}(\beta)=\frac{1}{48}\left(\frac{1}{\beta}-8 \beta\right)+\frac{1}{4} .
$$

\subsection{Implications for toric Calabi-Yau backgrounds}

The free energy $\mathcal{F}_{A}(Q ; \beta)$ of the refined topological string on the resolved conifold geometry, i.e., $\mathcal{O}(-1) \oplus \mathcal{O}(-1) \rightarrow \mathbb{P}^{1}$, is related to the refined deformed conifold free energy given by the integral (3.9) by identifying the Kähler parameter as $Q=e^{-\mu}$ and simply replacing the integral by a sum

$$
\int d t \rightarrow \sum_{d=1}^{\infty}
$$

This replacement originates from the sum over states of D0-brane charge $k \in \mathbb{Z}$ and mass $\sim \mu+2 \pi i k$, or, in M-theory language, from the extra state degeneracy due to momenta around the M-theory circle. It is natural to assume that a similar "quantization" as in (3.21) can be applied to the other Macdonald integrals as well. For $B_{N}$, we infer from (3.11)

$$
\mathcal{F}_{B}(Q ; \beta)=\mathcal{F}_{A}(Q ; 2 \beta)
$$

i.e., the resolved conifold free energy of $B_{N}$ type agrees with that of type $A_{N}$.

\footnotetext{
${ }^{6}$ It is interesting to note that this generating function looks very similar to the generating function for the massless hypermultiplet contribution occuring in $\mathrm{SU}(2)$ gauge theory on $\Omega$-deformed $A_{1}$ ALE space 42 .
} 
The $D_{N}$ ensemble is more interesting. Applying (3.21) to the (shifted) $D_{N}$ free energy (3.18), we obtain

$$
\mathcal{F}_{D}(Q ; \beta / 2)=\sum_{d=1}^{\infty} Q^{d} \frac{(q / t)^{d / 2}+(q / t)^{-d / 2}}{d\left(q^{d / 2}-q^{-d / 2}\right)\left(t^{-d}-t^{d}\right)}
$$

with the usual definitions $q:=e^{\sqrt{\beta} g_{s}}$ and $t:=e^{\frac{g_{s}}{\sqrt{\beta}}}$, as prediction for the refined free energy of $D_{N}$ type of the resolved conifold geometry. In particular, we have an even power only expansion in $g_{s}$ and the relation $\mathcal{F}_{D}(Q ; 1)=\mathcal{F}_{A}(Q ; 2)$ holds.

It is instructive to compare this result with the expectations based on a topological string orientifold interpretation. A convenient reference is the recent proposal [11] for the orientifolded and refined resolved conifold free energy. This proposal, which is obtained from an $S O(2 N)$ refined Chern-Simons/geometric transition point of view, reads,

$$
\mathcal{F}_{A}\left(t^{-1 / 2} Q ; \sqrt{2} g_{s}, 2 \beta\right)+\sum_{d=1}^{\infty} \frac{((q / t) Q)^{d / 2}}{t^{d / 2}-t^{-d / 2}} .
$$

(we have here exchanged $q \leftrightarrow t$ (corresponding to $\beta \leftrightarrow 1 / \beta$ ).) As observed in [11], the specialization of (3.24) to $\beta=1$ equals the free energy of an orientifold of the resolved conifold (acting either in fixed-point free [43], or in a real [44, 29] fashion). The second term in (3.24) can be understood as originating from the second term in (3.7) of the (unshifted) $D_{N}$ free energy, summing only over even D0 brane charge (up to a shift). It may also be seen as a brane placed at $-1 / 2 \log Q$ in the A-model geometry. Since the brane is localized in two space-time dimensions, it is exposed only to a single parameter of the $\Omega$-deformation (after a suitable redefinition of parameters).

The structure of the refined orientifold free energy (3.24) is consistent with the results of [34, where it was found that the free energy of the fixed-point free orientifold of $\mathcal{O}(-2) \oplus \mathcal{O}(-2) \rightarrow \mathbb{P}^{1} \times \mathbb{P}^{1}$ equals the refined free energy at $\beta=2$ (for this orientifold one has no open string sector).

We draw attention to the fact that, at $\beta=1$, the shift of $\log Q$ in the first term of (3.24) cancels the part of the sum in the second term coming from even $d$. That summation would be unusual for the $g_{s}$-odd sector of an orientifold. The cancellation is possible because the open string contribution is essentially a closed string period. On the other hand, this kind of comparison challenges the extrapolation of the proposed orientifold structure (3.24) to more general toric Calabi-Yau geometries, since a cancellation between a shift and an open string contribution is not possible in general. 
Instead, we propose to view $\mathcal{F}_{D}(Q ; \beta)$ of (3.23) as the refined free energy of type $D_{N}$ of the resolved conifold, independent of an orientifold interpretation. 7 Although for specific situations (such as the conifold or Dijkgraaf-Vafa type geometries, see section 5), when the orientifold contribution is a closed period, the (unshifted) $D_{N}$ free energy can be matched and interpreted at $\beta=1$ as an orientifold, this relation is not expected to persist in general.

Specifically, we propose to identify the theory of $D_{N}$ type with the orbifold branch in the $c=1$ moduli space, extending the identification of $A_{N}$ with the circle branch of $c=1$ [2]. The relation $\beta=R$ is the same on both branches. For a toric Calabi-Yau manifold, one may then use (3.18) as boundary condition on the holomorphic anomaly equation at the conifold point in moduli space in order to obtain predictions for the $D_{N}$ theory. We will not pursue this quite interesting toric direction further in this work, but rather stick to the Macdonald ensemble setting, where the correspondence with orientifolds holds provided we work with even potentials. This will give further evidence via explicit calculations that the deformation (2.4) away from $\beta=1$ is consistent.

\section{Gaussian ensembles: Correlators}

In order to evaluate perturbatively $\beta$-ensembles with multi-cut support we will need to evaluate normalized Gaussian correlators defined as

$$
C_{k_{1}, k_{2}, \ldots, k_{m}}^{(b, d)}(\beta):=\frac{\left\langle\prod_{i=1}^{m} S_{k_{i}}\right\rangle_{(b, d)}}{\langle 1\rangle_{(b, d)}}=\frac{1}{Z_{(b, d)}(\beta)} \int[d \lambda]\left|P_{(b, d)}(\lambda)\right|^{2 \beta} e^{-\frac{1}{2} \sum_{i=1}^{N} \lambda_{i}^{2}} \prod_{i=1}^{m} S_{k_{i}}
$$

with $S_{k}:=\sum_{i=1}^{N} \lambda_{i}^{k}$ and normalized via the Gaussian partition function $Z_{(b, d)}$ given in (2.1). Clearly,

$$
C_{0,0, \ldots, 0}^{(b, d)}(\beta)=N \times N \times \cdots \times N .
$$

Correlators with non-vanishing $k_{i}$ can be solved for recursively invoking the Wardidentities resulting from invariance under eigenvalue reparameterizations. For example, more recently this approach has been followed in the $A_{N}$ case with general $\beta$ in [18]. The generalization of this approach to the $B_{N}$ and $D_{N}$ case we are interested in is

\footnotetext{
${ }^{7}$ It is conceivable that this result (instead of (3.24) ) can be obtained also from the Chern-Simons point of view by appropriately incorporating the quantum shift (3.17) in the large- $N$ limit.
} 
straight-forward. The Ward identities read

$$
\sum_{k=1}^{N} \int[d \lambda] \partial_{\lambda_{k}}\left(\lambda_{k}^{n}\left(\Delta_{+}(\lambda)^{b+d} \Delta_{-}(\lambda) \prod_{i=1}^{N} \lambda_{i}^{b}\right)^{2 \beta} e^{-\frac{1}{2} \sum_{i=1}^{N} \lambda_{i}^{2}} S_{k_{1}} \ldots S_{k_{m}}\right)=0 .
$$

Acting with the derivative on each factor and expressing the resulting terms through the correlators (4.1) yields a recursive equation for them. For brevity, we will here explicitly state only the new contributions of $\Delta_{+}(\lambda)^{b+d}$ and $\prod_{i=1}^{N} \lambda_{i}^{b}$ which do not occur in the $A_{N}$ case. The remaining $A_{N}$ contributions can be deduced similarly.

Using,

$$
\sum_{k=1}^{N} \lambda_{k}^{n} \partial_{\lambda_{k}}\left(\prod_{i=1}^{N} \lambda_{i}^{b}\right)^{2 \beta}=2 \beta b S_{n-1}\left(\prod_{i=1}^{N} \lambda_{i}^{b}\right)^{2 \beta}
$$

the new contribution which only occurs for $B_{N}$ can be inferred to be simply given by

$$
2 \beta b C_{n-1, k_{1}, \ldots, k_{m}}^{(b, d)}
$$

The derivation of the contribution of $\Delta_{+}(\lambda)^{b+d}$ goes as follows. One rewrites

$$
\sum_{k=1}^{N} \lambda_{k}^{n} \partial_{\lambda_{k}}\left(\Delta_{+}(\lambda)^{2 \beta(b+d)}\right)=2 \beta(b+d) \Delta_{+}(\lambda)^{2 \beta(b+d)} \sum_{k=1}^{N} \lambda_{k}^{n} \partial_{\lambda_{k}} \log \Delta_{+}(\lambda),
$$

and deduces the identity

$$
2 \beta(b+d) \sum_{k=1}^{N} \lambda_{k}^{n} \partial_{\lambda_{k}} \log \Delta_{+}(\lambda)=(b+d) \beta \sum_{i \neq j} \frac{\lambda_{i}^{n}+\lambda_{j}^{n}}{\lambda_{i}+\lambda_{j}}=(b+d) \beta \sum_{i \neq j} \frac{\lambda_{i}^{n}-\left(-\lambda_{j}\right)^{n}}{\lambda_{i}-\left(-\lambda_{j}\right)} .
$$

Note that the second step in the above equality is only valid for $n$ odd. Fortunately, knowing only how to deal with the $n$ odd case is sufficient for our purposes. Finally, making use of the identity

$$
\sum_{i \neq j} \frac{\lambda_{i}^{n}-\left(-\lambda_{j}\right)^{n}}{\lambda_{i}-\left(-\lambda_{j}\right)}=\sum_{k=0}^{n-1} \sum_{i \neq j}(-1)^{k} \lambda_{i}^{n-k-1} \lambda_{j}^{k}=\sum_{k=0}^{n-1}(-1)^{k} S_{n-k-1} S_{k}-S_{n-1},
$$

one arrives at the contribution

$$
\beta(b+d) \sum_{k=0}^{n-1}(-1)^{k} C_{n-k-1, k, k_{1}, \ldots, k_{m}}^{(b, d)}-\beta(b+d) C_{n-1, k_{1}, \ldots, k_{m}}^{(b, d)} .
$$


Combining all terms, taking also the usual $A_{N}$ contribution into account (see for instance [18, 45]), the Ward identities (4.2) translate to the recursive relation

$$
\begin{aligned}
C_{n+1, k_{1}, k_{2}, \ldots, k_{m}}^{(b, d)}= & ((1-\beta) n+(b-d) \beta) C_{n-1, k_{1}, \ldots, k_{m}}^{(b, d)} \\
& +\beta \sum_{k=0}^{n-1}\left(1+(b+d)(-1)^{k}\right) C_{n-k-1, k, k_{1}, \ldots, k_{m}}^{(b, d)}+\sum_{j=1}^{m} k_{j} C_{k_{1}, \ldots, k_{j}+n-1, \ldots, k_{m}}^{(b, d)} .
\end{aligned}
$$

Note that the sole difference between the $B_{N}$ and $D_{N}$ case is a switch of sign of one of the terms entering the recursive relation.

As pointed out already above, in case of $B_{N}$ and $D_{N}$ the recursive relation is only valid for $n$ odd. However, the relation closes if furthermore all $k_{i}$ are even. Hence, it can be used to determine all correlators $C_{k_{1}, k_{2}, \ldots, k_{m}}^{(b, d)}$ with $k_{i}$ even. A few examples follow.

$$
\begin{aligned}
& C_{2}^{(0,1)}=N(1+2 \beta(N-1)), \quad C_{2}^{(1,0)}=N(1+2 \beta N), \\
& C_{2,2}^{(0,1)}=C_{2}^{(0,1)}\left(2+C_{2}^{(0,1)}\right), \quad C_{2,2}^{(1,0)}=C_{2}^{(1,0)}\left(2+C_{2}^{(1,0)}\right), \\
& C_{4}^{(0,1)}=C_{2}^{(0,1)}(3+4 \beta(N-1)), \quad C_{4}^{(1,0)}=C_{2}^{(1,0)}(3+\beta(4 N-2)) .
\end{aligned}
$$

One should note that while in the $A_{N}$ case with $\beta=1$ a generating function for the 1-point correlators $C_{n}^{(0,0)}$ is known [25] and there are also closed expressions for $\beta=1 / 2$ and $\beta=2$ [46], no such closed formula has been found for general $\beta$, nor for the $B_{N}$ and $D_{N}$ cases, so far. Nevertheless, we can make at least one general observation regarding the structure of the $C_{n}^{(b, d)}$ for $n$ even. Namely, the coefficients of the highest powers in $N$ appear to be always given in terms of the Catalan-numbers $C_{n}:=\frac{(2 n) !}{(n+1) ! n !}$, i.e.,

$$
\begin{gathered}
C_{2 n}^{(0,0)}(\beta)=C_{n} \beta^{n} N^{n+1}+\ldots, \quad C_{2 n}^{(1,0)}(\beta)=C_{n} \beta^{n} N^{n+1}+\ldots \\
C_{2 n}^{(0,1)}(\beta)=2^{n} C_{n} \beta^{n} N^{n+1}+\ldots
\end{gathered}
$$

\section{Multi-cut potentials: Perturbative calculation}

In this section we shall evaluate the eigenvalue ensembles (2.4) in a perturbative fashion via a saddle-point approximation, making use of the fact that for $g_{s} \rightarrow 0$ the eigenvalues $\lambda_{i}$ localize to the critical points of the potential $W(x)$. A detailed exposition of the perturbative calculation of the $\beta$-deformed $A_{N}$ partition function has been given in [18], as a generalization of the earlier $\beta=1$ works [19, 20, 17]. We will not repeat that discussion here, but focus on the new features that appear for $B_{N}$ and $D_{N}$ ensembles. 
We discuss only potentials with the symmetry $W(x)=W(-x)$. In particular, $W(x)$ is a polynomial of even degree.

\subsection{Saddle-point approximation}

Since the degree $d$ of $W(x)$ is even, we have an odd number $c=d-1$ of critical points. In particular, due to the $\mathbb{Z}_{2}$ symmetry of the potential there is one critical point at $\operatorname{Im}(x)=\operatorname{Re}(x)=0$. We denote the set of critical points as $\mu^{(k)}$ with $k \in$ $\left\{-\frac{c-1}{2}, \ldots, 0, \ldots, \frac{c-1}{2}\right\}$, hence $\mu^{(-k)}=-\mu^{(k)}$. The saddle-point approximation requires us to distribute the $N$ eigenvalues between the $c$ critical points. Let us denote the number of eigenvalues located around $\mu^{(k)}$ as $N_{k}$. In contrast to the $A_{N}$ case, we have to impose some additional constraints onto the eigenvalue distribution. This will allow the $B_{N}$ and $D_{N}$ ensembles to be dual to, both, $\mathcal{N}=2 S O / S p$ gauge theories with adjoint broken to $\mathcal{N}=1$ by a tree-level potential of the form $W(x)$ and to topological string orientifolds. From an orientifold point of view, it is more convenient to work in the quotient space perspective. In particular, for the eigenvalue ensembles this allows to avoid to deal with the interactions between "mirror" eigenvalues under the $\mathbb{Z}_{2}$ identification of cuts which the duality to orientifolds requires. Following [9], we implement the quotient into the eigenvalue ensemble by localizing the eigenvalues around the quotient set of critical points, i.e., we take the eigenvalues to be localized around $(c+1) / 2$ of the critical points such that

$$
N=N_{0}+\ldots+N_{\frac{c-1}{2}}
$$

The $A_{N}$ condition for consistency of the $\beta$-deformation of filling all cuts stated in the introduction then changes in the $B_{N} / D_{N}$ case to filling only the quotient set of cuts.

The partition function can then be evaluated by considering small fluctuations $y_{n}^{(k)}$, with $k \in\left\{0, \ldots, \frac{c-1}{2}\right\}$, around the critical points, i.e., we set

$$
\left(\lambda_{1}, \lambda_{2}, \ldots, \lambda_{N}\right)=\left(\mu^{(0)}+y_{1}^{(0)}, \ldots, \mu^{(0)}+y_{N_{0}}^{(0)}, \ldots\right) .
$$


Under this decomposition we have that

$$
\begin{aligned}
& \Delta_{-}(\lambda) \rightarrow \prod_{k=0}^{\frac{c-1}{2}} \prod_{i<j}^{N_{k}}\left(y_{i}^{(k)}-y_{j}^{(k)}\right) \prod_{0 \leq m<n \leq \frac{c-1}{2}} \prod_{i=1}^{N_{m}} \prod_{j=1}^{N_{n}}\left(\mu^{(m)}-\mu^{(n)}+y_{i}^{(m)}-y_{j}^{(n)}\right), \\
& \Delta_{+}(\lambda) \rightarrow \prod_{k=0}^{\frac{c-1}{2}} \prod_{i<j}^{N_{k}}\left(2 \mu^{(k)}+y_{i}^{(k)}+y_{j}^{(k)}\right) \prod_{0 \leq m<n \leq \frac{c-1}{2}} \prod_{i=1}^{N_{m}} \prod_{j=1}^{N_{n}}\left(\mu^{(m)}+\mu^{(n)}+y_{i}^{(m)}+y_{j}^{(n)}\right) .
\end{aligned}
$$

Hence,

$$
\left|P_{(b, d)}(\lambda)\right|^{2 \beta} \rightarrow\left(\prod_{k=1}^{\frac{c-1}{2}} \Delta_{-}\left(y^{(k)}\right) \Delta_{-}\left(y^{(0)}\right)\left(\Delta_{+}\left(y^{(0)}\right)\right)^{b+d} \prod_{i=1}^{N}\left(y_{i}^{(0)}\right)^{b}\right)^{2 \beta} \exp \mathcal{I}(y)
$$

with interaction term

$$
\begin{aligned}
\mathcal{I}(y)= & -2 \beta \sum_{0 \leq m<n \leq \frac{c-1}{2}} \sum_{l=1}^{\infty} \sum_{r=0}^{l} \frac{(-1)^{r}}{l\left(\mu^{(m)}-\mu^{(n)}\right)^{l}}\left(\begin{array}{c}
l \\
r
\end{array}\right) S_{r}^{(m)} S_{l-r}^{(n)} \\
& -\beta(b+d) \sum_{k=1}^{\frac{c-1}{2}} \sum_{l=1}^{\infty} \frac{(-1)^{l}}{2^{l} l\left(\mu^{(k)}\right)^{l}} \sum_{r=0}^{l}\left(\begin{array}{c}
l \\
r
\end{array}\right) S_{r}^{(k)} S_{l-r}^{(k)}+\beta(d-b) \sum_{k=1}^{\frac{c-1}{2}} \sum_{l=1}^{\infty} \frac{(-1)^{l}}{l\left(\mu^{(k)}\right)^{l}} S_{l}^{(k)} \\
& -2 \beta(b+d) \sum_{0 \leq m<n \leq \frac{c-1}{2}} \sum_{l=1}^{\infty} \frac{(-1)^{l}}{l\left(\mu^{(m)}+\mu^{(n)}\right)^{l}} \sum_{r=0}^{l}\left(\begin{array}{l}
l \\
r
\end{array}\right) S_{r}^{(m)} S_{l-r}^{(n)}+\text { const. . }
\end{aligned}
$$

The potential decomposes as

$$
\sum_{i=1}^{N} W\left(\lambda_{i}\right) \rightarrow \sum_{k=0}^{\frac{c-1}{2}} \sum_{n=1}^{\infty} \frac{\left(\partial^{n} W\right)\left(\mu^{(k)}\right)}{n !} S_{n}^{(k)}+\text { const. . }
$$

Note that the $B_{N}$, respectively $D_{N}$ ensemble decomposes in the saddle-point approximation into $(c-1) / 2 A_{N}$ and a single $B_{N}$, respectively $D_{N}$ eigenvalue ensembles, which are coupled via the interaction term $\mathcal{I}(y)$, as expected. Hence, after an appropriate $g_{s}$ dependent redefinition of $S_{i}^{(m)}$, and expansion in $g_{s}$ to bring down powers of $S_{i}^{(m)}$, the partition function of the eigenvalue ensemble (2.4) reduces in the saddle-point approximation to a sum over Gaussian correlators which are determinable via the results of section 4. Due to the normalization of the Gaussian correlators, the resulting partition function has to be supplemented for each cut by a factor of $Z_{\mathcal{G}}(\beta)$ (defined in (2.1)) with $\mathcal{G}$ either $B_{N}$ or $D_{N}$ for the fixed cut and else $A_{N}$. 


\subsection{Examples: $B_{N}$ and $D_{N}$ quartic}

Let us now consider an example in more detail. The simplest non-trivial $\mathbb{Z}_{2}$ symmetric example is given by the quartic potential

$$
W(\lambda)=\frac{\beta}{g_{s}} g\left(\frac{1}{4} \lambda^{4}-\frac{\delta^{2}}{2} \lambda^{2}\right) .
$$

Clearly, $W(-\lambda)=W(\lambda)$ and the set of critical points $\mu^{(k)}$ consists of

$$
\mu^{(-1)}=-\delta, \quad \mu^{(0)}=0, \quad \mu^{(1)}=\delta
$$

hence possesses a three-cut structure. The partition functions $Z_{(b, d)}$ for $A_{N}, B_{N}$ and $D_{N}$ measure can be obtained in a perturbative fashion as outlined in the previous section. While one has to fill in the $A_{N}$ case all three cuts as discussed in the introduction, for $B_{N}$ and $D_{N}$ one has to fill only two of the cuts in order to incorporate the $\mathbb{Z}_{2}$ quotient as mentioned in section 5.1 .

For the $A_{N}$ case, let us just quote the relevant observation without giving any further details. Namely, we observe that if we fill all three-cuts, the disk sector $\left(g_{s}^{-1}\right)$ of the corresponding free energy is a combination of closed periods, i.e.,

$$
\tilde{\mathcal{F}}_{A}^{(1 / 2)}=\frac{1}{2}\left(1-\frac{1}{\beta}\right)\left(\frac{\partial \mathcal{F}_{A}^{(0)}}{\partial \tilde{S}_{-1}}+\frac{\partial \mathcal{F}_{A}^{(0)}}{\partial \tilde{S}_{0}}+\frac{\partial \mathcal{F}_{A}^{(0)}}{\partial \tilde{S}_{1}}\right)
$$

where as usual $\tilde{S}_{i}:=N_{i} g_{s}$. This indicates that one has to perform the additional quantum shifts

$$
S_{i}:=\left(N_{i}-\frac{1}{2}\left(1-\frac{1}{\beta}\right)\right) g_{s}
$$

in the large $N$ limit. Indeed, after the shifts, one obtains an expansion into only even powers of $g_{s}$ of the free energy $\mathcal{F}_{A}$, as is necessary for a well-defined BPS index interpretation of the corresponding partition function.

Let us now consider the $B_{N}$ and $D_{N}$ cases. For the quartic potential (5.5), the measure specializes to

$$
\left[d y^{(0)}\right]\left[d y^{(1)}\right]\left(\Delta_{-}\left(y^{(1)}\right) \Delta_{-}\left(y^{(0)}\right)\left(\Delta_{+}\left(y^{(0)}\right)\right)^{b+d} \prod_{i=1}^{N}\left(y_{i}^{(0)}\right)^{b}\right)^{2 \beta}
$$


the interaction term (5.3) reads

$$
\begin{aligned}
\mathcal{I}(y)= & -2 \beta \sum_{l=1}^{\infty} \sum_{r=0}^{l} \frac{(-1)^{l}}{l \delta^{l}}\left(\begin{array}{l}
l \\
r
\end{array}\right)\left(1+(b+d)(-1)^{r}\right) S_{r}^{(0)} S_{l-r}^{(1)} \\
& -\beta(b+d) \sum_{l=1}^{\infty} \frac{(-1)^{l}}{2^{l} l \delta^{l}} \sum_{r=0}^{l}\left(\begin{array}{l}
l \\
r
\end{array}\right) S_{r}^{(1)} S_{l-r}^{(1)}+\beta(d-b) \sum_{l=1}^{\infty} \frac{(-1)^{l}}{l \delta^{l}} S_{l}^{(1)}+\text { const. },
\end{aligned}
$$

and the potential contribution (5.4) is given by

$$
\mathcal{W}(y)=-\sum_{n=1}^{\infty} \frac{1}{n !}\left(\left(\partial^{n} W\right)(0) S_{n}^{(0)}+\left(\partial^{n} W\right)(\delta) S_{n}^{(1)}\right)
$$

After performing the rescalings

$$
S_{n}^{(0)} \rightarrow\left(-\frac{g_{s}}{\beta g \delta^{2}}\right)^{n / 2} S_{n}^{(0)}, \quad S_{n}^{(1)} \rightarrow\left(\frac{g_{s}}{2 \beta g \delta^{2}}\right)^{n / 2} S_{n}^{(1)},
$$

the partition functions can be expanded in $g_{s}$ and reduce to a sum over Gaussian correlators, which one can efficiently calculate following section 4. For the reader's convenience, the explicit free energies to some lower order in $g_{s}$ are given in appendix A. Defining

$$
S_{0}=2 N_{0} g_{s}, \quad S_{1}=N_{1} g_{s}
$$

we obtain

$$
\tilde{\mathcal{F}}_{B}^{(0)}\left(S_{0}, S_{1}\right)=\tilde{\mathcal{F}}_{D}^{(0)}\left(S_{0}, S_{1}\right)=\frac{1}{2} \tilde{\mathcal{F}}_{A}^{(0)}\left(S_{0}, S_{1}, S_{-1}=S_{1}\right)
$$

which is the expected tree-level result from a topological string orientifold perspective. The first order open string corrections $\left(g_{s}^{-1}\right)$ read

$$
\begin{aligned}
& \tilde{\mathcal{F}}_{B}^{(1 / 2)}=\frac{1}{2}\left(2-\frac{1}{\beta}\right) \frac{\partial \mathcal{F}_{B}^{(0)}}{\partial \tilde{S}_{0}}+\frac{1}{2}\left(1-\frac{1}{\beta}\right) \frac{\partial \mathcal{F}_{B}^{(1)}}{\partial \tilde{S}_{1}} \\
& \tilde{\mathcal{F}}_{D}^{(1 / 2)}=-\frac{1}{2 \beta} \frac{\partial \mathcal{F}_{D}^{(0)}}{\partial \tilde{S}_{0}}+\frac{1}{2}\left(1-\frac{1}{\beta}\right) \frac{\partial \mathcal{F}_{D}^{(1)}}{\partial \tilde{S}_{1}}
\end{aligned}
$$

and are combinations of closed string periods. Note that for $\beta=1$ we have that $\tilde{\mathcal{F}}_{B}^{(1 / 2)}=$ $-\tilde{\mathcal{F}}_{D}^{(1 / 2)}=-\frac{1}{4} \partial_{S_{0}} \mathcal{F}_{A}\left(S_{0}, S_{1}, S_{-1}=S_{1}\right)$, confirming the earlier results of [8, 47, 48, 9]. In the dual topological string orientifold, the sign difference translates into the two possible choices of charge of the orientifold fixed-plane. Similar as for the $A_{N}$ case, the 
order $g_{s}^{-1}$ given in (5.10) suggests to perform the additional quantum shifts as in (5.6) such that

$$
\mathcal{F}_{B}^{(1 / 2)}=-\frac{1}{2} \frac{\partial \tilde{\mathcal{F}}_{A}^{(0)}\left(S_{0}, S_{1}, S_{-1}=S_{1}\right)}{\partial \tilde{S}_{0}}, \quad \tilde{\mathcal{F}}_{D}^{(1 / 2)}=\frac{1}{2} \frac{\partial \tilde{\mathcal{F}}_{A}^{(0)}\left(S_{0}, S_{1}, S_{-1}=S_{1}\right)}{\partial \tilde{S}_{0}}
$$

and the relation $\mathcal{F}_{B}^{(1 / 2)}=-\mathcal{F}_{D}^{(1 / 2)}$ continues to hold under the $\beta$-deformation. In particular, the open string contribution at order $g_{s}^{-1}$ is independent of $\beta$. However, for higher orders in $g_{s}$, one has that generally

$$
\mathcal{F}_{B}^{(g>1 / 2)}(\beta) \neq \mathcal{F}_{D}^{(g>1 / 2)}(\beta),
$$

and equality (up to overall sign) only for $\beta=1$. Since the open string contribution is trivial (i.e., it is a closed period), it is more convenient to shift away the complete open string contribution such that $\mathcal{F}_{B}^{(g / 2)}=\mathcal{F}_{D}^{(g / 2)}=0$, for $g$ odd. Hence, in this specific gauge the free energies possess an expansion into even powers of $g_{s}$ only. If necessary, the open string contribution can be easily reinstated via performing a reverse shift. The main advantage of this shift is that it allows us to utilize the usual holomorphic anomaly of [33] instead of the extended holomorphic of [37] to reproduce the $B_{N}$ and $D_{N}$ partition functions in the B-model. On a technical level, the former is easier to deal with. However, we like to stress that the latter is more general, since it is expected to capture the partition function independent of any shift of parameters, similar as observed at hand of gauge theory in [34, 35].

Finally, let us comment on the Nekrasov-Shatashvili limit of the free energies (in the gauge with an even power $g_{s}$ expansion). In our parameterization the limit of [5] corresponds to

$$
\mathcal{W}_{\mathcal{G}}^{(g)}:=\lim _{\beta \rightarrow 0} \beta^{g} \mathcal{F}_{\mathcal{G}}^{(g)}(\beta) .
$$

From our explicit computations we observe that

$$
\frac{1}{2} \mathcal{W}_{A}^{(g)}=\mathcal{W}_{B}^{(g)}=\mathcal{W}_{D}^{(g)}
$$

A similar non-uniqueness property of the limit has been already observed at hand of gauge theory on ALE space in [42, and is in fact as expected. This is because, since $g_{s} \rightarrow 0$ (in order to keep $\hbar:=\frac{g_{s}}{\sqrt{\beta}}$ fixed, $c f$., (1.1)), the Nekrasov-Shatashvili limit, and hence $\mathcal{W}^{(g)}$, is intrinsically of tree-level nature. More specifically, the limit corresponds to a (semi-classical limit of a) quantization of the spectral curve of the respective eigenvalue ensemble, following [3]. Since the spectral curves of the $A_{N}, B_{N}$ and $D_{N}$ 
ensembles (under a proper $\mathbb{Z}_{2}$ identification of the $A_{N}$ spectral curve) are identical ( $c f$., (5.9) ), so should be the quantization thereof. The relation (5.13) shows that this is indeed the case.

\section{B-model verification of $B_{N}$ and $D_{N}$ quartic}

\subsection{Tree-level geometry}

The dual tree-level geometry of the quartic eigenvalue ensemble with $B_{N}$ and $D_{N}$ measure with $\beta=1$ has been discussed already in the literature to some extent ( $c f$. , [49, 8, 9]). Since the power of $\beta$ is only relevant at one-loop and beyond, we essentially can borrow the known tree-level results.

The periods of the dual geometry of the eigenvalue ensemble with potential $W(x)$ of the form (5.5) are given in terms of the periods of the hyperelliptic curve

$$
y=M(x) \sqrt{\sigma(x)},
$$

with

$$
M(x)=g, \quad \sigma(x)=\frac{1}{g^{2}}\left(W^{\prime}(x)^{2}+f(x)\right),
$$

and where $f(x)$ is a degree two polynomial. In particular, the moment function $M(x)$ is a constant because we fill all cuts. For the quartic, the curve (6.1) is of genus two. The effective one-form of the dual geometry reads

$$
\omega=y d x
$$

which we express in terms of the six branch points $x_{i}$ of the curve as

$$
\omega=g \sqrt{\prod_{i=1}^{6}\left(x-x_{i}\right)} d x .
$$

The cuts are chosen to be $\left[x_{1}, x_{2}\right],\left[x_{3}, x_{4}\right]$ and $\left[x_{5}, x_{6}\right]$ on the real axis. Imposing the $\mathbb{Z}_{2}$ symmetry under $x \rightarrow-x$ of the $x$-plane (this requires that $f(x)$ is even, i.e., $f(x)=$ $b_{2} x^{2}+b_{0}$ with $b_{i}$ parameterizing the complex structure) leads to the identification $x_{1} \leftrightarrow-x_{6}, x_{2} \leftrightarrow-x_{5}$ and $x_{3} \leftrightarrow-x_{4}$, yielding the one-form

$$
\omega=g \sqrt{\left(x^{2}-x_{1}^{2}\right)\left(x^{2}-x_{2}^{2}\right)\left(x^{2}-x_{3}^{2}\right)} d x .
$$

The $\mathbb{Z}_{2}$ symmetric x-plane of the geometry is illustrated in figure 1. 


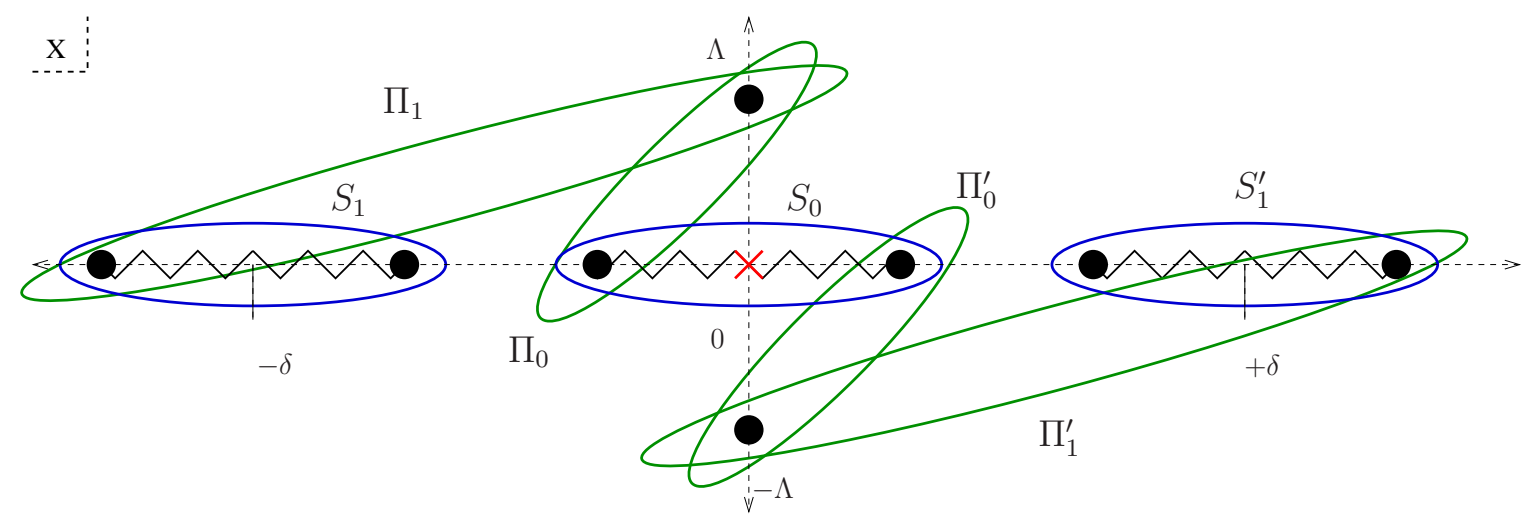

Figure 1: The symmetric $x$-plane of the quartic with cuts, period contours and reflection symmetry indicated (covering space perspective).

One should note that if one adjusts such that $b_{2}=0$, the curve (6.1) takes the form of the Seiberg-Witten curve of four dimensional $\mathcal{N}=2 S U(3)$ gauge theory, with choice of Coulomb parameters $a_{1}=-a_{2}$ and $a_{3}=0$ (the zeroes of $W^{\prime}(x)$ correspond to the $a_{i}$ ), which can also be matched to the curve of $S p(2)$. It would be interesting to extract the gauge theory gauge coupling and 1-loop gravitational correction following [50, 20] and see if one can match to a $\Omega$-deformed gauge theory, as is the case for the cubic and $\Omega$-deformed $S U(2)$ [3]. However, since $a_{3}=0$, we are not on the Coloumb branch, and things are expected to be a bit more tricky. Therefore we will not follow this rather interesting direction further in this work.

Comparison with the one-form (6.3) expressed via (6.2) gives the relation

$$
\delta^{2}=\frac{1}{2}\left(x_{1}^{2}+x_{2}^{2}+x_{3}^{2}\right) .
$$

In order to explicitly calculate the period integrals, it is useful to change variables to

$$
z_{0}=x_{3}, \quad z_{1}=\frac{1}{2}\left(x_{2}-x_{1}\right), \quad \mathcal{I}=\frac{1}{2}\left(x_{1}+x_{2}\right)
$$

Hence, we set

$$
x_{3}=z_{0}, \quad x_{1}=\mathcal{I}-z_{1}, \quad x_{2}=\mathcal{I}+z_{1} .
$$

Using (6.4), we can infer for $\mathcal{I}$ the relation

$$
\mathcal{I}= \pm \sqrt{\delta^{2}-\frac{1}{2} z_{0}^{2}-z_{1}^{2}}
$$


The discriminant $\Delta$ of the algebraic curve (6.1) reads in the $z_{i}$ coordinates (6.5)

$$
\Delta=4 z_{0}^{2} z_{1}^{4} \Delta_{1}^{2} \Delta_{2}^{2} \Delta_{3}^{4},
$$

with components

$$
\begin{aligned}
& \Delta_{1}=z_{0}^{2}+2 z_{1}^{2}-2 \delta^{2}, \quad \Delta_{2}=z_{0}^{2}+4 z_{1}^{2}-2 \delta^{2}, \\
& \Delta_{3}=9 z_{0}^{4}+4 z_{0}^{2}\left(2 z_{1}^{2}-3 \delta^{2}\right)+4\left(-2 z_{1}^{2}+\delta^{2}\right)^{2} .
\end{aligned}
$$

In particular, we have that $\Delta_{1}=-2 \mathcal{I}^{2}$.

The A-periods of the curve (6.1) with one-form (6.3) are taken to be

$$
S_{0}=\frac{1}{2 \pi i} \int_{-x_{3}}^{x_{3}} \omega, \quad S_{1}=\frac{1}{2 \pi i} \int_{x_{2}}^{x_{1}} \omega .
$$

It is not hard to explicitly evaluate the integrals in the coordinates (6.5) for small $z_{i}$. The first few terms read

$$
\begin{aligned}
& S_{0}\left(z_{i}\right)=-\frac{g \delta^{2}}{4} z_{0}^{2}+\frac{g}{2} z_{0}^{2} z_{1}^{2}+\frac{3 g}{16} z_{0}^{4}+\frac{g}{8 \delta^{2}} z_{0}^{4} z_{1}^{2}+\frac{g}{4 \delta^{4}} z_{0}^{4} z_{1}^{4}+\frac{g}{8 \delta^{4}} z_{0}^{6} z_{1}^{2}+\ldots, \\
& S_{1}\left(z_{i}\right)=\frac{g \delta^{2}}{2} z_{1}^{2}-\frac{g}{2} z_{0}^{2} z_{1}^{2}-\frac{g}{2} z_{1}^{4}-\frac{g}{16 \delta^{2}} z_{0}^{4} z_{1}^{2}-\frac{g}{8 \delta^{4}} z_{0}^{4} z_{1}^{4}-\frac{g}{16 \delta^{4}} z_{0}^{6} z_{1}^{2}+\ldots,
\end{aligned}
$$

Note that the two periods are related via the identity

$$
S_{1}+\frac{1}{2} S_{0}=\frac{g}{32}\left(z_{0}^{2}-4 z_{1}^{2}\right)\left(3 z_{0}^{2}+4 z_{1}^{2}-4 \delta^{2}\right) .
$$

Inversion of (6.9) yields the so-called mirror maps $z_{i}\left(S_{i}\right)$.

Similarly, it is not hard to explicitly evaluate the B-periods

$$
\Pi_{0}=\frac{1}{2} \int_{x_{3}}^{\Lambda} \omega, \quad \Pi_{1}=\int_{x_{1}}^{\Lambda} \omega,
$$

where $\Lambda \rightarrow \infty$ is a cutoff. Note the additional factor of $1 / 2$ we introduced for $\Pi_{0}$. Its origin can be most easily seen at hand of figure 1 . The period $\Pi_{0}$ (without the $1 / 2$ ) in the quotient space corresponds in the covering space actually to $2 \Pi_{0}$ and not just $\Pi_{0}$.

We obtain in terms of the flat coordinates $S_{i}$ for the B-periods

$$
\begin{aligned}
\Pi_{0}\left(S_{i}\right)= & \frac{g}{8}\left(\Lambda^{2}-2 \delta^{2}\right) \Lambda^{2}-\frac{1}{2} S_{0} \log g-2\left(S_{1}+\frac{1}{2} S_{0}\right) \log \Lambda \\
& +2\left(S_{1}-\frac{1}{2} S_{0}\right) \log \delta+P_{0}\left(S_{0}, S_{1}\right), \\
\Pi_{1}\left(S_{i}\right)= & \frac{g}{4}\left(\delta^{2}-\Lambda^{2}\right)^{2}-4\left(S_{1}+\frac{1}{2} S_{0}\right) \log \Lambda+2\left(S_{1}+S_{0}\right) \log \delta+S_{1} \log \left(\frac{2}{g \delta^{2}}\right) \\
& +P_{1}\left(S_{0}, S_{1}\right),
\end{aligned}
$$


with $P_{i}\left(S_{0}, S_{1}\right)=S_{i} \log S_{i}+\sum_{n, m \geq 0} \frac{c_{i}(n, m)}{\left(g \delta^{2}\right)^{n+m-1}} S_{0}^{n} S_{1}^{m}$ and $c_{i}(n, m)$ constants .

Invoking the usual special geometry relation

$$
\left.\partial_{S_{i}} \mathcal{F}^{(0)}=\Pi_{i}\left(S_{0}, S_{1}\right)\right)
$$

the prepotential $\mathcal{F}^{(0)}$ can be determined, and indeed matches the results of section 5.2. Note that the $P_{i}\left(S_{0}, S_{1}\right)$ can be expressed in terms of the flat-coordinate Yukawa couplings $C_{S_{i} S_{j} S_{k}}$ as $P_{i}\left(S_{0}, S_{1}\right)=\int d S_{i} d S_{i} C_{S_{i} S_{i} S_{i}}$. Closed expressions for the Yukawa couplings in $z_{i}$ coordinates, i.e., $C_{z_{i} z_{j} z_{k}}:=D_{z_{i}} D_{z_{j}} D_{z_{k}} \mathcal{F}^{(0)}\left(z_{i}\right)$ with $D_{z_{i}}$ denoting the covariant derivative ( $c f .$, [33]), can be found to be

$$
\begin{aligned}
& C_{z_{0} z_{0} z_{0}}=-\frac{z_{0}\left(9 z_{0}^{6}+6 z_{0}^{4}\left(-5+z_{1}^{2}\right)+8\left(1-2 z_{1}^{2}\right)^{2}\left(-1+z_{1}^{2}\right)+4 z_{0}^{2}\left(7-8 z_{1}^{2}+4 z_{1}^{4}\right)\right)}{32 \mathcal{I}^{2}}, \\
& C_{z_{1} z_{1} z_{1}}=-\frac{z_{1}\left(3 z_{0}^{6}+8\left(-1+2 z_{1}^{2}\right)^{3}+2 z_{0}^{4}\left(-7+10 z_{1}^{2}\right)+4 z_{0}^{2}\left(5-16 z_{1}^{2}+12 z_{1}^{4}\right)\right.}{4 \mathcal{I}^{2}}, \\
& C_{z_{0} z_{0} z_{1}}=\frac{z_{1} z_{0}^{2}\left(-2+3 z_{0}^{2}\right)\left(-2+z_{0}^{2}+4 z_{1}^{2}\right)}{8 \mathcal{I}^{2}}, \\
& C_{z_{0} z_{1} z_{1}}=\frac{z_{0} z_{1}^{2}\left(z_{0}^{4}-4\left(1-2 z_{1}^{2}\right)^{2}\right)}{4 \mathcal{I}^{2}} .
\end{aligned}
$$

The remaining couplings follow by symmetry. We have also set for simplicity $g=\delta=1$.

\subsection{One-loop}

Having the tree-level data at hand, it is straight-forward to evaluate the solution to the 1-loop holomorphic anomaly equation of [51]

$$
\mathcal{F}^{(1)}(z ; \beta)=\frac{1}{2} \log \operatorname{det} G+a^{(1)}(z ; \beta)
$$

with $G_{i j}:=\partial_{S_{i}} z_{j}$ and $a^{(1)}(z ; \beta)$ denoting the 1-loop holomorphic ambiguity. The ambiguity can be parameterized in terms of the discriminant loci $\Delta_{i}$ given in (6.7) as

$$
a^{(1)}(z ; \beta)=\nu_{0} \log z_{0}+\nu_{1} \log z_{1}+\kappa_{1} \log \Delta_{1}+\kappa_{2} \log \Delta_{2}+\kappa_{3} \log \Delta_{3} .
$$

From the eigenvalue ensemble results of section 5.2 we deduce that under fixing parameters $\nu_{i}$ and $\kappa_{i}$ in the $B_{N}$ case to

$$
\begin{aligned}
& \nu_{0}=\frac{1}{24}\left(\frac{1}{\beta}+4 \beta\right)-\frac{1}{2}, \quad \nu_{1}=\frac{1}{12}\left(\frac{1}{\beta}+\beta\right)-\frac{1}{2}, \\
& \kappa_{1}=\nu_{0}, \quad \kappa_{2}=\frac{1}{2} \nu_{1}+\frac{1}{4}, \quad \kappa_{3}=\nu_{1},
\end{aligned}
$$


and for $D_{N}$ to

$$
\begin{aligned}
& \nu_{0}=\frac{1}{24}\left(\frac{1}{\beta}-8 \beta\right), \quad \nu_{1}=\frac{1}{12}\left(\frac{1}{\beta}+\beta\right)-\frac{1}{2}, \\
& \kappa_{1}=\nu_{0}, \quad \kappa_{2}=\frac{1}{2} \nu_{1}+\frac{1}{4}, \quad \kappa_{3}=\nu_{1},
\end{aligned}
$$

the previous results can be reproduced. We observe that the ambiguities for both cases differ in general only in $\nu_{0}$. Further, note that only in the special case of $\beta=1$ and in the Nekrasov-Shatashvili limit $(\beta \rightarrow 0)$ the $\nu_{0}$ of both cases are equal and the relation

$$
\mathcal{F}_{B}^{(1)}=\mathcal{F}_{D}^{(1)}
$$

holds, as expected from the relations (3.19) and (5.13). For general $\beta$ this equality will not hold anymore. Also note that in the Nekrasov-Shatashvili limit (5.12) the 1-loop amplitude (6.13) becomes purely holomorphic, i.e.,

$$
\mathcal{W}^{(1)}(z)=\frac{1}{24} \log z_{0}+\frac{1}{12} \log z_{1}+\frac{1}{24} \log \left(\Delta_{1} \Delta_{2}\right)+\frac{1}{12} \log \Delta_{3} .
$$

The reason being that in this limit the 1-loop anomaly equation reduces to

$$
\bar{\partial}_{\bar{i}} \partial_{j} \mathcal{W}^{(1)}(z, \bar{z})=0
$$

To conclude this section, it is interesting to compare the $\nu_{0}$ coefficients we found to

the corresponding $\Psi_{\mathcal{G}}^{(0)}$ coefficients of section $[3$, given in (3.12) and (3.20). Up to an addition of $1 / 2$ they match. We attribute the additional $1 / 2$ to an artifact of our expansion at the 1-loop level and/or to the chosen parameterization. Mainly because the same mismatch by $1 / 2$ occurs for $\nu_{1}$, which should be equal to $\Psi_{A}^{(0)}$.

\section{Conclusion}

In this work we initiated the study of $\beta$-ensembles with $B_{N}$ and $D_{N}$ measure beyond tree-level. For that purpose, we generalized the calculation of the $\beta$-deformed $A_{N}$ partition function of [18], which makes use of a saddle-point approximation and Ward identities, to the $B_{N}$ and $D_{N}$ cases. At hand of the quartic, we found that the resulting free energies possess an expansion into even powers of $g_{s}$ only, under a specific choice of 't Hooft parameters. This is as expected, since the $g_{s}^{-1}$ sector is a closed period and should be removable via an appropriate shift of parameters following [35]. The absence of an odd sector in $g_{s}$ allowed us to invoke the usual holomorphic anomaly equation to 
reproduce the 1-loop sector $\left(g_{s}^{0}\right)$ of the quartic, albeit with new boundary conditions (holomorphic ambiguity) which have not appeared (to our knowledge) before. The boundary conditions are related to a large $N$ expansion of the Macdonald integral. We expect that the higher genus coefficients of the Macdonald integral expansions will provide boundary conditions for the higher loop amplitudes expanded near some of the other points in moduli space. However, so far we have not pushed the holomorphic anomaly calculation for the quartic beyond genus one, and it would be interesting to do so. Our results indicate that the $\beta$-deformation of the $B_{N}$ and $D_{N}$ ensembles, which for $\beta=1$ correspond to topological string orientifolds, is consistent.

The Gaussian integrals also allowed us to extrapolate the $B_{N}$ and $D_{N}$ ensembles to toric settings. We found that the $D_{N}$ case (under an appropriate shift) should correspond to the topological string with boundary conditions at the conifold point provided by the orbifold branch of the $c=1$ string. The $B_{N}$ case corresponds to the usual circle branch, similar to the standard refined topological string related to $A_{N}$ ensemble. In the toric setting, our results indicate that the equivalence between $B_{N} / D_{N}$ and topological string orientifolds is not general. Rather the $c=1$ moduli space appears to yield an independent deformation space of topological string theories, with only accidental correspondence to topological string orientifolds for specific simple geometries.

It seems likely that one may also explore ensembles with $E_{6}, E_{7}$ and $E_{8}$ type measure in a similar fashion. Presumably, these ensembles are related to the three discrete points in $c=1$ moduli space (and should not possess a consistent $\beta$-deformation).

\section{Acknowledgments}

We like to thank S-Y. D. Shih for related discussions. We thank the KITP for hospitality during the INTEGRAL11 program where this work was started. D.K. thanks McGill University, and J.W. thanks CERN-PH TH Division for hospitality during completion of this work. The work of D.K. has been supported by a Simons fellowship, and by the Berkeley Center for Theoretical Physics. The work of J.W. is supported in part by the Canada Research Chair program and an NSERC discovery grant. This work was also supported in part by DARPA under Grant No. HR0011-091-0015, and the NSF under Grant No. PHY05-51164. 


\section{A Free energies}

The explicit results for the perturbative expansion of the free energies of the $B_{N}$ and $D_{N}$ eigenvalue ensemble with quartic potential discussed in section 5.2 are given below. Note that the expressions given do not take the normalization of (4.1) into account. The additional contribution from the normalization to the shifted free energy is given in terms of an asymptotic expansion of the respective Macdonald integral given in (2.2).

$$
\begin{aligned}
& \tilde{\mathcal{F}}_{B}\left(N_{i}, \beta\right)=-\frac{g_{s}}{4 \delta^{4} \beta}\left(8 N_{0}^{3} \beta^{2}-2 N_{0}^{2} \beta\left(2 \beta\left(8 N_{1}+1\right)-5\right)+N_{0}\left(8 \beta^{2}\left(N_{1}-3\right) N_{1}+\beta\left(8 N_{1}-2\right)+3\right)+(2 \beta-1) N_{1}\left(2 \beta\left(N_{1}-2\right)+3\right)\right) \\
& +\frac{g_{s}^{2}}{4 g^{2} \delta^{8} \beta^{2}}\left(36 N_{0}^{4} \beta^{3}-4 N_{0}^{3} \beta^{2}\left(2 \beta\left(28 N_{1}+5\right)-19\right)+N_{0}^{2} \beta\left(12 \beta^{2}\left(12 N_{1}^{2}-16 N_{1}+1\right)+\beta\left(24 N_{1}-50\right)+53\right)\right. \\
& +N_{0}\left(-8 \beta^{3} N_{1}\left(2 N_{1}^{2}-15 N_{1}+19\right)+\beta^{2}\left(-48 N_{1}^{2}+200 N_{1}+6\right)-\beta\left(88 N_{1}+15\right)+12\right)-(2 \beta-1) N_{1}\left(4 \beta^{2}\left(N_{1}^{2}-5 N_{1}+5\right)\right. \\
& \left.\left.+\beta\left(15 N_{1}-29\right)+12\right)\right) \\
& -\frac{g_{s}^{3}}{12 g^{3} \delta^{12} \beta^{3}}\left(864 N_{0}^{5} \beta^{4}-8 N_{0}^{4} \beta^{3}\left(4 \beta\left(233 N_{1}+49\right)-331\right)+8 N_{0}^{3} \beta^{2}\left(8 \beta^{2}\left(131 N_{1}^{2}-102 N_{1}+16\right)-4 \beta\left(29 N_{1}+103\right)+383\right)\right. \\
& -4 N_{0}^{2} \beta\left(4 \beta^{2}\left(165 N_{1}^{2}-888 N_{1}-80\right)+4 \beta^{3}\left(152 N_{1}^{3}-558 N_{1}^{2}+640 N_{1}+15\right)+2 \beta\left(965 N_{1}+286\right)-393\right)+N_{0}\left(8 \beta ^ { 2 } \left(419 N_{1}^{2}\right.\right. \\
& \left.\left.-798 N_{1}+48\right)+32 \beta^{4} N_{1}\left(5 N_{1}^{3}-66 N_{1}^{2}+206 N_{1}-165\right)+8 \beta^{3}\left(112 N_{1}^{3}-1050 N_{1}^{2}+1208 N_{1}-15\right)+12 \beta\left(88 N_{1}-43\right)+297\right) \\
& \left.+(2 \beta-1) B\left(4 \beta^{2}\left(66 N_{1}^{2}-314 N_{1}+307\right)+8 \beta^{3}\left(5 N_{1}^{3}-44 N_{1}^{2}+108 N_{1}-74\right)+6 \beta\left(86 N_{1}-163\right)+297\right)\right) \\
& -\frac{g_{s}^{4}}{8 g^{4} \delta^{16} \beta^{4}}\left(-6048 N_{0}^{6} \beta^{5}+16 N_{0}^{5} \beta^{4}\left(\beta\left(4232 N_{1}+982\right)-1549\right)-4 N_{0}^{4} \beta^{3}\left(4 \beta^{2}\left(7088 N_{1}^{2}-3492 N_{1}+1033\right)-2 \beta\left(3596 N_{1}\right.\right.\right. \\
& +5973)+10325)+8 N_{0}^{3} \beta^{2}\left(\beta^{2}\left(3232 N_{1}^{2}-29640 N_{1}-4326\right)+16 \beta^{3}\left(456 N_{1}^{3}-1088 N_{1}^{2}+1275 N_{1}+65\right)+\beta\left(18132 N_{1}\right.\right. \\
& +6853)-4350)-N_{0}^{2} \beta\left(4 \beta^{2}\left(27696 N_{1}^{2}-29580 N_{1}+5981\right)+32 \beta^{3}\left(740 N_{1}^{3}-7254 N_{1}^{2}+6111 N_{1}-325\right)+16 \beta^{4}\left(628 N_{1}^{4}\right.\right. \\
& \left.\left.-4216 N_{1}^{3}+11172 N_{1}^{2}-7232 N_{1}+105\right)+48 \beta\left(131 N_{1}-581\right)+14691\right)+N_{0}\left(48 \beta^{2}\left(515 N_{1}^{2}-1808 N_{1}-112\right)\right. \\
& +16 \beta^{3}\left(1474 N_{1}^{3}-7593 N_{1}^{2}+9782 N_{1}+195\right)+32 \beta^{5} N_{1}\left(14 N_{1}^{4}-279 N_{1}^{3}+1522 N_{1}^{2}-2956 N_{1}+1769\right)+8 \beta^{4}\left(488 N_{1}^{4}\right. \\
& \left.\left.-7680 N_{1}^{3}+22104 N_{1}^{2}-17872 N_{1}-105\right)+3 \beta\left(7520 N_{1}+1743\right)-2448\right)+(2 \beta-1) N_{1}\left(6 \beta^{2}\left(632 N_{1}^{2}-2905 N_{1}+2799\right)\right. \\
& \left.\left.+4 \beta^{3}\left(279 N_{1}^{3}-2318 N_{1}^{2}+5492 N_{1}-3695\right)+16 \beta^{4}\left(7 N_{1}^{4}-93 N_{1}^{3}+398 N_{1}^{2}-658 N_{1}+353\right)+\beta\left(5229 N_{1}-9795\right)+2448\right)\right) \\
& -\frac{g_{s}^{5}}{10 g^{5} \delta^{20} \beta^{5}}\left(93312 N_{0}^{7} \beta^{6}-288 N_{0}^{6} \beta^{5}\left(2 \beta\left(2252 N_{1}+559\right)-1685\right)+16 N_{0}^{5} \beta^{4}\left(24 \beta^{2}\left(7711 N_{1}^{2}-2423 N_{1}+1250\right)\right.\right. \\
& \left.-2 \beta\left(31728 N_{1}+40813\right)+67259\right)-40 N_{0}^{4} \beta^{3}\left(4 \beta^{2}\left(1605 N_{1}^{2}-47267 N_{1}-9059\right)+12 \beta^{3}\left(4784 N_{1}^{3}-8246 N_{1}^{2}+10442 N_{1}\right.\right. \\
& \left.+803)+2 \beta\left(62029 N_{1}+26899\right)-32463\right)+4 N_{0}^{3} \beta^{2}\left(40 \beta^{2}\left(35489 N_{1}^{2}-20156 N_{1}+10312\right)+40 \beta^{3}\left(5544 N_{1}^{3}-66014 N_{1}^{2}\right.\right. \\
& \left.\left.+39818 N_{1}-5027\right)+48 \beta^{4}\left(3670 N_{1}^{4}-16580 N_{1}^{3}+41550 N_{1}^{2}-21780 N_{1}+863\right)-30 \beta\left(4710 N_{1}+14933\right)+223489\right) \\
& -2 N_{0}^{2} \beta\left(60 \beta^{2}\left(10356 N_{1}^{2}-51699 N_{1}-6938\right)+20 \beta^{3}\left(54900 N_{1}^{3}-186600 N_{1}^{2}+252646 N_{1}+13849\right)+40 \beta^{4}\left(4038 N_{1}^{4}\right.\right. \\
& \left.-61004 N_{1}^{3}+137454 N_{1}^{2}-109848 N_{1}-2589\right)+16 \beta^{5}\left(2568 N_{1}^{5}-26610 N_{1}^{4}+120040 N_{1}^{3}-192810 N_{1}^{2}+109832 N_{1}+945\right)+ \\
& \left.\beta\left(982802 N_{1}+373894\right)-164865\right)+N_{0}\left(4 \beta^{2}\left(246049 N_{1}^{2}-415992 N_{1}+38745\right)+120 \beta^{3}\left(4782 N_{1}^{3}-34959 N_{1}^{2}+35464 N_{1}\right.\right. \\
& -1034)+8 \beta^{4}\left(35850 N_{1}^{4}-334320 N_{1}^{3}+989930 N_{1}^{2}-715820 N_{1}+7767\right)+64 \beta^{6} N_{1}\left(42 N_{1}^{5}-1158 N_{1}^{4}+9500 N_{1}^{3}-31960 N_{1}^{2}\right. \\
& \left.+45679 N_{1}-22355\right)+16 \beta^{5}\left(2064 N_{1}^{5}-47710 N_{1}^{4}+240920 N_{1}^{3}-460650 N_{1}^{2}+265116 N_{1}-945\right)+30 \beta\left(6880 N_{1}-4111\right) \\
& +50139)+(2 \beta-1) N_{1}\left(4 \beta^{2}\left(27945 N_{1}^{2}-125282 N_{1}+119563\right)+8 \beta^{3}\left(5895 N_{1}^{3}-46950 N_{1}^{2}+108328 N_{1}-71927\right)\right. \\
& +8 \beta^{4}\left(1158 N_{1}^{4}-14460 N_{1}^{3}+59320 N_{1}^{2}-95329 N_{1}+50293\right)+32 \beta^{5}\left(21 N_{1}^{5}-386 N_{1}^{4}+2480 N_{1}^{3}-7080 N_{1}^{2}+9025 N_{1}\right. \\
& \left.\left.-4081)+6 \beta\left(20555 N_{1}-38242\right)+50139\right)\right) \\
& +\mathcal{O}\left(g_{s}^{6}\right) \text {, }
\end{aligned}
$$




$$
\begin{aligned}
& \tilde{\mathcal{F}}_{D}\left(N_{i}, \beta\right)=\frac{g_{s}}{4 g \delta^{4} \beta}\left(-9 N_{0}^{3} \beta^{2}+N_{1}\left(3+2\left(-1+N_{1}\right) \beta\right)+2 N_{0}^{2} \beta\left(-5+8\left(1+2 N_{1}\right) \beta\right)-N_{0}\left(3+2\left(-5+4 N_{1}\right) \beta+8\left(1+N_{1}+N_{1}^{2}\right) \beta^{2}\right)\right) \\
& +\frac{g_{s}^{2}}{4 g^{2} \delta^{8} \beta^{2}}\left(36 N_{0}^{4} \beta^{3}-4 N_{0}^{3} \beta^{2}\left(-19+28\left(1+2 N_{1}\right) \beta\right)+N_{1}\left(12+15\left(-1+N_{1}\right) \beta+2\left(3-5 N_{1}+2 N_{1}^{2}\right) \beta^{2}\right)\right. \\
& +N_{0}^{2} \beta\left(53+2\left(-77+12 N_{1}\right) \beta+4\left(29+36 N_{1}+36 N_{1}^{2}\right) \beta^{2}\right)-N_{0}\left(-12+\left(53+88 N_{1}\right) \beta+2\left(-39-68 N_{1}+24 N_{1}^{2}\right) \beta^{2}\right. \\
& \left.+8\left(5+11 N_{1}+3 N_{1}^{2}+2 N_{1}^{3}\right) \beta^{3}\right) \\
& +\frac{g_{s}^{3}}{12 g^{3} \delta^{12} \beta^{3}}\left(-864 N_{0}^{5} \beta^{4}+8 N_{0}^{4} \beta^{3}\left(466 \beta\left(2 N_{1}+1\right)-331\right)-8 N_{0}^{3} \beta^{2}\left(4 \beta^{2}\left(262 N_{1}^{2}+262 N_{1}+189\right)-4 \beta\left(29 N_{1}+260\right)+383\right)\right. \\
& +4 N_{0}^{2} \beta\left(20 \beta^{2}\left(33 N_{1}^{2}-174 N_{1}-109\right)+4 \beta^{3}\left(152 N_{1}^{3}+228 N_{1}^{2}+622 N_{1}+273\right)+2 \beta\left(965 N_{1}+781\right)-393\right) \\
& -N_{0}\left(8 \beta^{2}\left(419 N_{1}^{2}+98 N_{1}+398\right)+8 \beta^{3}\left(112 N_{1}^{3}-546 N_{1}^{2}-412 N_{1}-381\right)+32 \beta^{4}\left(5 N_{1}^{4}+10 N_{1}^{3}+80 N_{1}^{2}+75 N_{1}+37\right)\right. \\
& \left.\left.+12 \beta\left(88 N_{1}-131\right)+297\right)+N_{1}\left(24 \beta^{2}\left(11 N_{1}^{2}-27 N_{1}+16\right)+8 \beta^{3}\left(5 N_{1}^{3}-22 N_{1}^{2}+32 N_{1}-15\right)+516 \beta\left(N_{1}-1\right)+297\right)\right) \\
& +\frac{g_{s}^{4}}{8 g^{4} \delta^{16} \beta^{4}}\left(6048 N_{0}^{6} \beta^{5}-16 N_{0}^{5} \beta^{4}\left(2116 \beta\left(2 N_{1}+1\right)-1549\right)+4 N_{0}^{4} \beta^{3}\left(4 \beta^{2}\left(7088 N_{1}^{2}+7088 N_{1}+4771\right)-2 \beta\left(3596 N_{1}+13449\right)\right.\right. \\
& +10325)-8 N_{0}^{3} \beta^{2}\left(\beta^{2}\left(3232 N_{1}^{2}-34536 N_{1}-21990\right)+24 \beta^{3}\left(304 N_{1}^{3}+456 N_{1}^{2}+1054 N_{1}+451\right)+3 \beta\left(6044 N_{1}+5433\right)\right. \\
& -4350)+N_{0}^{2} \beta\left(12 \beta^{2}\left(9232 N_{1}^{2}+7404 N_{1}+11455\right)+32 \beta^{3}\left(740 N_{1}^{3}-5286 N_{1}^{2}-5271 N_{1}-4010\right)+16 \beta^{4}\left(628 N_{1}^{4}+1256 N_{1}^{3}\right.\right. \\
& \left.\left.+7236 N_{1}^{2}+6608 N_{1}+3085\right)+48 \beta\left(131 N_{1}-1483\right)+14691\right)-N_{0}\left(48 \beta^{2}\left(515 N_{1}^{2}-1296 N_{1}-758\right)+16 \beta^{3}\left(1474 N_{1}^{3}\right.\right. \\
& \left.-789 N_{1}^{2}+5814 N_{1}+3023\right)+8 \beta^{4}\left(488 N_{1}^{4}-3552 N_{1}^{3}-2496 N_{1}^{2}-9680 N_{1}-4401\right)+32 \beta^{5}\left(14 N_{1}^{5}+35 N_{1}^{4}+490 N_{1}^{3}\right. \\
& \left.\left.+700 N_{1}^{2}+937 N_{1}+353\right)+3 \beta\left(7520 N_{1}+4897\right)-2448\right)+N_{1}\left(48 \beta^{2}\left(79 N_{1}^{2}-191 N_{1}+112\right)+12 \beta^{3}\left(93 N_{1}^{3}-398 N_{1}^{2}\right.\right. \\
& \left.\left.\left.+565 N_{1}-260\right)+8 \beta^{4}\left(14 N_{1}^{4}-93 N_{1}^{3}+234 N_{1}^{2}-260 N_{1}+105\right)+5229 \beta\left(N_{1}-1\right)+2448\right)\right) \\
& +\frac{g_{s}^{5}}{10 g^{5} \delta^{20} \beta^{5}}\left(-93312 N_{0}^{7} \beta^{6}+288 N_{0}^{6} \beta^{5}\left(2252 \beta\left(2 N_{1}+1\right)-1685\right)-16 N_{0}^{5} \beta^{4}\left(8 \beta^{2}\left(23133 N_{1}^{2}+23133 N_{1}+14833\right)\right.\right. \\
& \left.-2 \beta\left(31728 N_{1}+85145\right)+67259\right)+40 N_{0}^{4} \beta^{3}\left(4 \beta^{2}\left(1605 N_{1}^{2}-60559 N_{1}-38558\right)+4 \beta^{3}\left(14352 N_{1}^{3}+21528 N_{1}^{2}+\right.\right. \\
& \left.\left.44618 N_{1}+18721\right)+2 \beta\left(62029 N_{1}+58538\right)-32463\right)-4 N_{0}^{3} \beta^{2}\left(20 \beta^{2}\left(70978 N_{1}^{2}+79294 N_{1}+96033\right)+80 \beta^{3}\left(2772 N_{1}^{3}\right.\right. \\
& \left.-29003 N_{1}^{2}-31778 N_{1}-21998\right)+32 \beta^{4}\left(5505 N_{1}^{4}+11010 N_{1}^{3}+52315 N_{1}^{2}+46810 N_{1}+20967\right)-30 \beta\left(4710 N_{1}+34231\right) \\
& +223489)+2 N_{0}^{2} \beta\left(60 \beta^{2}\left(10356 N_{1}^{2}-51393 N_{1}-36176\right)+20 \beta^{3}\left(54900 N_{1}^{3}+22896 N_{1}^{2}+252052 N_{1}+140723\right)\right. \\
& +40 \beta^{4}\left(4038 N_{1}^{4}-38428 N_{1}^{3}-45138 N_{1}^{2}-110094 N_{1}-50559\right)+16 \beta^{5}\left(2568 N_{1}^{5}+6420 N_{1}^{4}+63600 N_{1}^{3}+88980 N_{1}^{2}\right. \\
& \left.\left.+110042 N_{1}+40405\right)+\beta\left(982802 N_{1}+915556\right)-164865\right)-N_{0}\left(\beta^{2}\left(984196 N_{1}^{2}+99616 N_{1}+937156\right)+120 \beta^{3}\left(4782 N_{1}^{3}\right.\right. \\
& \left.-19317 N_{1}^{2}-10208 N_{1}-12766\right)+8 \beta^{4}\left(35850 N_{1}^{4}-57660 N_{1}^{3}+373880 N_{1}^{2}+274580 N_{1}+194147\right)+16 \beta^{5}\left(2064 N_{1}^{5}\right. \\
& \left.-20630 N_{1}^{4}-9820 N_{1}^{3}-140460 N_{1}^{2}-115344 N_{1}-58455\right)+64 \beta^{6}\left(42 N_{1}^{6}+126 N_{1}^{5}+2730 N_{1}^{4}+5250 N_{1}^{3}+13309 N_{1}^{2}\right. \\
& \left.\left.+10705 N_{1}+4081\right)+30 \beta\left(6880 N_{1}-10991\right)+50139\right)+N_{1}\left(540 \beta^{2}\left(207 N_{1}^{2}-494 N_{1}+287\right)+120 \beta^{3}\left(393 N_{1}^{3}-1643 N_{1}^{2}\right.\right. \\
& \left.+2284 N_{1}-1034\right)+24 \beta^{4}\left(386 N_{1}^{4}-2480 N_{1}^{3}+6050 N_{1}^{2}-6545 N_{1}+2589\right)+16 \beta^{5}\left(42 N_{1}^{5}-386 N_{1}^{4}+1450 N_{1}^{3}-2750 N_{1}^{2}\right. \\
& \left.\left.\left.+2589 N_{1}-945\right)+123330 \beta\left(N_{1}-1\right)+50139\right)\right) \\
& +\mathcal{O}\left(g_{s}^{6}\right) \text {. }
\end{aligned}
$$




\section{References}

[1] R. Dijkgraaf and C. Vafa, "Matrix models, topological strings, and supersymmetric gauge theories," Nucl. Phys. B 644 (2002) 3 arXiv:hep-th/0206255.

[2] R. Dijkgraaf and C. Vafa, "Toda Theories, Matrix Models, Topological Strings, and N=2 Gauge Systems," arXiv:0909.2453 [hep-th].

[3] M. Aganagic, M. C. N. Cheng, R. Dijkgraaf, D. Krefl and C. Vafa, "Quantum Geometry of Refined Topological Strings," arXiv:1105.0630 [hep-th].

[4] L. F. Alday, D. Gaiotto and Y. Tachikawa, "Liouville Correlation Functions from Four-dimensional Gauge Theories," Lett. Math. Phys. 91 (2010) 167 arXiv:0906.3219 [hep-th]].

[5] N. A. Nekrasov and S. L. Shatashvili, "Quantization of Integrable Systems and Four Dimensional Gauge Theories," arXiv:0908.4052 [hep-th].

[6] M. Aganagic and S. Shakirov, "Knot Homology from Refined Chern-Simons Theory," arXiv:1105.5117 [hep-th]

[7] D. Ghoshal and C. Vafa, "C = 1 string as the topological theory of the conifold," Nucl. Phys. B 453, 121 (1995) arXiv:hep-th/9506122.

[8] S. K. Ashok, R. Corrado, N. Halmagyi, K. D. Kennaway and C. Romelsberger, "Unoriented strings, loop equations, and $\mathrm{N}=1$ superpotentials from matrix models," Phys. Rev. D 67 (2003) 086004 [arXiv:hep-th/0211291].

[9] K. A. Intriligator, P. Kraus, A. V. Ryzhov, M. Shigemori and C. Vafa, "On low rank classical groups in string theory, gauge theory and matrix models," Nucl. Phys. B 682 (2004) 45 [arXiv:hep-th/0311181].

[10] N. Nekrasov and S. Shadchin, "ABCD of instantons," Commun. Math. Phys. 252 (2004) 359 arXiv:hep-th/0404225.

[11] M. Aganagic and K. Schaeffer, "Orientifolds and the Refined Topological String," arXiv:1202.4456 [hep-th],

[12] K. Landsteiner, C. I. Lazaroiu and R. Tatar, "(Anti)symmetric matter and superpotentials from IIB orientifolds," JHEP 0311 (2003) 044 [arXiv:hep-th/0306236].

[13] K. Landsteiner and C. I. Lazaroiu, "On $\mathrm{Sp}(0)$ factors and orientifolds," Phys. Lett. B 588 (2004) 210 arXiv:hep-th/0310111.

[14] A. Brini, M. Marino and S. Stevan, "The Uses of the refined matrix model recursion," arXiv:1010.1210 [hep-th],

[15] V. Bouchard, A. Klemm, M. Marino and S. Pasquetti, "Remodeling the B-model," Commun. Math. Phys. 287 (2009) 117 arXiv:0709.1453 [hep-th].

[16] M. Marino, "Open string amplitudes and large order behavior in topological string theory," JHEP 0803 (2008) 060 arXiv:hep-th/0612127. 
[17] A. S. Alexandrov, A. Mironov and A. Morozov, "Partition functions of matrix models as the first special functions of string theory. 1. Finite size Hermitean one matrix model," Int. J. Mod. Phys. A 19 (2004) 4127 [Teor. Mat. Fiz. 142 (2005) 419] arXiv:hep-th/0310113.

[18] A. Morozov and S. Shakirov, "The matrix model version of AGT conjecture and CIV-DV prepotential," JHEP 1008 (2010) 066 arXiv:1004.2917 [hep-th]].

[19] M. Aganagic, A. Klemm, M. Marino and C. Vafa, "Matrix model as a mirror of ChernSimons theory," JHEP 0402 (2004) 010 arXiv:hep-th/0211098.

[20] A. Klemm, M. Marino and S. Theisen, "Gravitational corrections in supersymmetric gauge theory and matrix models," JHEP 0303 (2003) 051 arXiv:hep-th/0211216.

[21] I. G. Macdonald, "Some Conjectures for Root Systems," SIAM J. Math. Anal., Vol. 13, No. 6, November 1982

[22] E. M. Opdam, "Some applications of hypergeometric shift operators," Invent. Math. 98, 275-282, 1989

[23] E. M. Opdam, "Dunkl operators, Bessel functions and the discriminant of a finite Coexter group," Compositio Math. 85, 333-373, 1993

[24] M. Aganagic, R. Dijkgraaf, A. Klemm, M. Marino and C. Vafa, "Topological strings and integrable hierarchies," Commun. Math. Phys. 261 (2006) 451 arXiv:hep-th/0312085].

[25] J. Harer and D. Zagier, "The Euler Characteristic of the Moduli Space of Curves," Inv. Math. 85 (1986) 457-485

[26] L. Chekhov and A. Zabrodin, "A Critical matrix model for nonoriented string," Mod. Phys. Lett. A 6 (1991) 3143.

[27] I. P. Goulden, J. L. Harer and D. M. Jackson, "A geometric parametrization for the virtual Euler characteristic of the moduli space of real and complex algebraic curves," Trans. Am. Math. Soc. 353, 4405 (2001)

[28] H. Ooguri and C. Vafa, "World sheet derivation of a large N duality," Nucl. Phys. B 641 (2002) 3 arXiv:hep-th/0205297.

[29] D. Krefl, S. Pasquetti and J. Walcher, "The Real Topological Vertex at Work," Nucl. Phys. B 833 (2010) 153 arXiv:0909.1324 [hep-th]].

[30] D. Krefl, "Wall Crossing Phenomenology of Orientifolds," arXiv:1001.5031 [hep-th].

[31] D. J. Gross and I. R. Klebanov, "One-dimensional String Theory On A Circle," Nucl. Phys. B 344 (1990) 475.

[32] M. -x. Huang, A. Klemm and S. Quackenbush, "Topological string theory on compact Calabi-Yau: Modularity and boundary conditions," Lect. Notes Phys. 757 (2009) 45 arXiv:hep-th/0612125.

[33] M. Bershadsky, S. Cecotti, H. Ooguri and C. Vafa, "Kodaira-Spencer theory of gravity and exact results for quantum string amplitudes," Commun. Math. Phys. 165 (1994) 311 arXiv:hep-th/9309140. 
[34] D. Krefl and J. Walcher, "Extended Holomorphic Anomaly in Gauge Theory," Lett. Math. Phys. 95 (2011) 67 [arXiv:1007.0263 [hep-th]].

[35] D. Krefl and J. Walcher, "Shift versus Extension in Refined Partition Functions," arXiv:1010.2635 [hep-th].

[36] M. -x. Huang and A. Klemm, "Direct integration for general $\Omega$ backgrounds," arXiv:1009.1126 [hep-th].

[37] J. Walcher, "Extended holomorphic anomaly and loop amplitudes in open topological string," Nucl. Phys. B 817 (2009) 167 arXiv:0705.4098 [hep-th]].

[38] D. Krefl and J. Walcher, "The Real Topological String on a local Calabi-Yau," arXiv:0902.0616 [hep-th].

[39] P. H. Ginsparg, "Curiosities at c = 1," Nucl. Phys. B 295 (1988) 153.

[40] R. Gopakumar and C. Vafa, "Topological gravity as large N topological gauge theory," Adv. Theor. Math. Phys. 2 (1998) 413 arXiv:hep-th/9802016.

[41] D. Ghoshal, D. P. Jatkar and S. Mukhi, "Kleinian singularities and the ground ring of C=1 string theory," Nucl. Phys. B 395 (1993) 144 [arXiv:hep-th/9206080].

[42] D. Krefl and S. -Y. D. Shih, "Holomorphic Anomaly in Gauge Theory on ALE space," arXiv:1112.2718 [hep-th],

[43] B. S. Acharya, M. Aganagic, K. Hori and C. Vafa, "Orientifolds, mirror symmetry and superpotentials," arXiv:hep-th/0202208.

[44] J. Walcher, "Evidence for Tadpole Cancellation in the Topological String," arXiv:0712.2775 [hep-th],

[45] A. Mironov, A. Morozov, A. Popolitov and S. .Shakirov, "Resolvents and Seiberg-Witten representation for Gaussian beta-ensemble," arXiv:1103.5470 [hep-th].

[46] I. P. Goulden and D. M. Jackson "Maps in Locally Orientable Surfaces and Integrals over Real Symmetric Surfaces," Can. J. Math. Vol. 49(5), 1997 pp. 865-882

[47] H. Ita, H. Nieder and Y. Oz, "Perturbative computation of glueball superpotentials for SO(N) and USp(N)," JHEP 0301 (2003) 018 [hep-th/0211261].

[48] R. A. Janik and N. A. Obers, "SO(N) superpotential, Seiberg-Witten curves and loop equations," Phys. Lett. B 553 (2003) 309 [hep-th/0212069].

[49] H. Fuji and Y. Ookouchi, "Confining phase superpotentials for SO / Sp gauge theories via geometric transition," JHEP 0302 (2003) 028 [arXiv:hep-th/0205301].

[50] R. Dijkgraaf, S. Gukov, V. A. Kazakov and C. Vafa, "Perturbative analysis of gauged matrix models," Phys. Rev. D 68 (2003) 045007 arXiv:hep-th/0210238.

[51] M. Bershadsky, S. Cecotti, H. Ooguri and C. Vafa, "Holomorphic anomalies in topological field theories," Nucl. Phys. B 405 (1993) 279 arXiv:hep-th/9302103. 